\title{
On the abundance discrepancy problem in $\mathrm{H}$ II regions. ${ }^{1}$
}

\author{
Jorge García-Rojas \\ Instituto de Astrofísica de Canarias, E-38200, La Laguna, Tenerife, Spain \\ jogarcia@ll.iac.es \\ and \\ César Esteban \\ Instituto de Astrofísica de Canarias, E-38200, La Laguna, Tenerife, Spain \\ cel@ll.iac.es
}

\begin{abstract}
The origin of the abundance discrepancy -i.e. the fact that abundances derived from recombination lines are larger than those from collisionaly excited lines- is one of the key problems in the physics of photoionized nebula. In this work, we analize and discuss data for a sample of Galactic and extragalactic H II regions where this abundance discrepancy has been determined. We find that the abundance discrepancy factor (ADF) is fairly constant and of the order of 2 in all the available sample of H II regions. This is a rather different behaviour than that observed in planetary nebulae, where the ADF shows a much wider range of values. We do not find correlations between the $\mathrm{ADF}$ and the $\mathrm{O} / \mathrm{H}$, $\mathrm{O}^{++} / \mathrm{H}^{+}$ratios, the ionization degree, $T_{e}(\mathrm{High}), T_{e}(\mathrm{Low}) / T_{e}(\mathrm{High}), \mathrm{FWHM}$, and the effective temperature of the main ionizing stars within the observational uncertainties. These results indicate that whatever mechanism is producing the abundance discrepancy in H II regions it does not substantially depend on those nebular parameters. On the contrary, the ADF seems to be slightly dependent on the excitation energy, a fact that is consistent with the predictions of the classical temperature fluctuations paradigm. Finally, we obtain that $T_{e}$ values obtained from $\mathrm{O}$ II recombination lines in $\mathrm{H}$ II regions are in agreement with those obtained from collisionally excited line ratios, a behaviour that is again different from that observed in planetary nebulae. These similar temperature
\end{abstract}

\footnotetext{
${ }^{1}$ Based on observations collected at the European Southern Observatory, Chile, proposals number ESO 68.C-0149(A) and ESO 70.C-0008(A).
} 
determinations are in contradiction with the predictions of the model based on the presence of chemically inhomogeneous clumps but are consistent with the temperature fluctuations paradigm. We conclude that all the indications suggest that the physical mechanism responsible of the abundance discrepancy in H II regions and planetary nebulae are different.

Subject headings: line:identification. ISM:abundances-H II regions.

\section{Introduction}

H II regions are essential tools for the study of the chemical composition and star formation in the Universe, specially in the extragalactic domain. It is necessary to be confident that our traditional techniques for deriving chemical abundances in ionized nebulae (based on the analysis of intensity ratios of colisionally excited lines, CELs) provide the real values.

Forty years ago, Peimbert (1967) characterized the temperature structure of a gaseous nebula to a second order by two parameters: the average temperature, $T_{0}$ and the mean square temperature fluctuation, $t^{2}$. At least, two independent methods of determining the electron temperature $T_{\mathrm{e}}$ of the nebula -with different weights in the high and low temperature regions- are necessary to derive $T_{0}$ and $t^{2}$. In their observations, Peimbert (1967) found that the temperature derived from the Balmer discontinuity, using the intensity ratio $\mathrm{BJ} / I(\mathrm{H} \beta)$ where BJ is the value of the Balmer jump, was systematically lower than that derived from the [O III] 4363/5007 CELs ratio. This result has been corroborated by several subsequent

works (see e.g. García-Rojas et al. 2006, and references therein) and we will refer to this problem as the temperature discrepancy.

Later, Peimbert \& Costerd 1969) claimed that, in the presence of temperature variations over the observed volume of the nebula, the gaseous abundances derived from the analysis of intensity ratios of CELs are underestimated if these temperature fluctuations are not considered. This is due to the strong dependence of the intensity of those lines on the assumed $T_{\mathrm{e}}$. This possibility is annoying, considering that the analysis of CELs is the standard method for deriving ionic abundances in ionized nebulae. On the other hand, intensity ratios of optical recombination lines (ORLs) are almost independent of the temperature structure of the nebula, and could be more appropiate to derive the "real" abundances of the nebulae. In the last years, several measurements of ORLs on H II regions and planetary nebulae (PNe) have been done, and it has been found that abundance determinations from ORLs are systematically higher than those obtained using CELs, independently of the ion considered (see e.g. Liu 2006; García-Rojas et al. 2006, and references therein). This is usually known 
as the abundance discrepancy problem.

Thanks to the fast technological progress -related to the development of large telescopes and better detectors- and to the significant improvement in the understanding of the recombination theory for multielectronic atomic systems, many works have been dedicated to understand the origin of the abundance and temperature discrepancies. During the last years, it has been confirmed that both effects are real, and that they are not related neither to systematic biases in the observations nor in the basic atomic physics. In addition, it has been found that the two dichotomies can be intimately related (see Liu et al. 2000).

The high surface brightness of the brightest PNe has permitted to detect many ORLs of heavy elements in these objects. The pioneering work of Wyse (1942) in NGC 7009, was the first in calling the attention on the different $\mathrm{O}^{++}$abundance obtained from O II ORLs and [O III] CELs. Later, Aller \& Menze (1945) and Torres-Peimbert \& Peimbert (1977) also remarked the excess of carbon observed in some $\mathrm{PNe}$ when calculating the $\mathrm{C}^{++}$abundance from C II ORLs. Since the availability of the $I U E$ satellite, carbon abundances from UV CELs could be determined, obtaining abundances systematically smaller than those derived from ORLs (see e.g. Harrington et al. 1980; Kaler 1986; Rola \& Stasińska 1994; Peimbert et al. 1995). During the last decade many efforts have been done to understand the physical conditions under which the different types of spectral lines are formed in PNe. In particular, the use of medium-large aperture telescopes, has allowed that the catalogue of detected O II and C II ORLs in PNe has increased spectacularly in the last years. The works dedicated to the study of the abundance discrepancy and/or the temperature structure in PNe are fairly numerous (e.g. Garnett \& Dinerstein 2001; Mathis et al. 1998; Liu et al. 2001, 2006, 2004; Peimbert et al. 2004; Robertson-Tessi \& Garnett 2005; Tsamis et al. 2004; Wesson et al. 2005, and references therein). The data collected in these works, indicate that the $t^{2}$ parameter shows an extraordinary range of variation in $\mathrm{PNe}$, reaching very high values in some objects. Liu et al. (2006), found values of $t^{2} \sim 0.14$ and 0.18 in Hf 2-2, from the abundance and temperature discrepancies, respectively. On the other hand, Rubin et al. (2002), from HST image and spectroscopy of PN NGC 7009, found that the $T_{\mathrm{e}}$ map of this nebula was practically uniform and that $t^{2}$ throughout the nebula on the plane of the sky was small $\left(t^{2}{ }_{A}=0.0035\right)$. In spite of the low $t^{2}$ value found, it could not completely be discarded the existence of temperature fluctuations throughout the line of sight that could account for the observed abundance discrepancies in this object (see Liu et al. 1995).

The abundance discrepancy is commonly quantified using the so-called abundance discrepancy factor (ADF), that is defined as:

$$
\operatorname{ADF}\left(\mathrm{X}^{+i}\right)=\left(\mathrm{X}^{+i} / \mathrm{H}^{+}\right)_{\text {ORLs }} /\left(\mathrm{X}^{+i} / \mathrm{H}^{+}\right)_{\mathrm{CELs}}
$$


Liu et al. (2001) found a correlation between the ADF and the difference between $T_{\mathrm{e}}([\mathrm{O} \mathrm{III}])$ and $T_{\mathrm{e}}(\mathrm{Bac})$. Qualitatively, this correlation is consistent with the existence of temperature fluctuations, although these authors argue that abundance and temperature discrepancies are qualitatively better explained on the basis of a chemically inhomogenous or two-phase model. Liu et al. (2000) analized the emission line spectrum of the PN NGC 6153, and concluded that the nebula contains a component of the ionized gas previously unknown -cold and very metal-rich-. Later works have supported this model, sustaining it mainly in the fact that the [O III] fine structure far infrared (FIR) lines at $\lambda \lambda 52,88 \mu \mathrm{m}$-which have very low excitation energies, of the order of $1000 \mathrm{~K}$, and are almost insensible to the variations of $T_{\mathrm{e}}$ - give ionic abundances comparable to the ones obtained from UV and optical CELs. These results are not compatible with the temperature fluctuations paradigm (see Liu 2006, and references therein), but can be explained by a two-phase model (see below).

The observational features that allow us to determine the $t^{2}$ parameter (ORLs, H I recombination continua) are much more difficult to be accurately measured in $\mathrm{H}$ II regions than in PNe. The low surface brightness of most of these objects has caused that, until a few years ago, the $\mathrm{H}$ II regions sample with $t^{2}$ determinations available has been limited to a few Galactic H II regions: the Orion nebula (Torres-Peimbert et al. 1980; Osterbrock et al. 1992; Peimbert et al. 1993a; Esteban et al. 1998; Rubin et al. 2003), M8 (Peimbert et al. 1993b; Esteban et al. 1999b) and M17 (Peimbert et al. 1992; Esteban et al. 1999a; Tsamis et al. 2003), and to a few extragalactic H II regions (González-Delgado et al. 1994; Esteban et al. 2002). In the last four years, this sample has been extended with the works of Tsamis et al. (2003) on an additional Galactic H II region (NGC 3576) and 3 extragalactic ones (1 in the Large Magellanic Cloud, LMC and 2 in the Small Magellanic Cloud, SMC); Peimbert (2003) on 30 Doradus; Peimbert et al. 2005) on 2 NGC 6822 H II regions; and López-Sánchez et al. (2007) on the starburst galaxy NGC 5253. Aditionally, O'Dell et al. (2003) made a direct estimation of $t^{2}$ in the plane of the sky of the Orion nebula from deep WFC/HST images, and making some geometrical considerations found a value of $t^{2}=0.028 \pm 0.006$, consistent with previous results $\left(t^{2}\right.$ between 0.020 and 0.030, see e.g. Esteban et al. 2004, and references therein). Finally, our group has obtained and analyzed extremely deep echelle spectra of a sample of 8 Galactic H II regions (Esteban et al. 2004; García-Rojas et al. 2004, 2005, 2006, 2007). In 4 of them (M16, M20, S 311 and NGC 3603), C II and O II ORLs were detected, for the first time; in other two regions (Orion nebula and NGC 3576) Ne II ORLs were also detected for the first time.

On the contrary, some puzzling results for the temperature fluctuations hypothesis have been recently found for some extragalactic H II regions. On the one hand, Hägele et al. (2006) have measured $T_{\mathrm{e}}(\mathrm{Bac})$ in three compact $\mathrm{H}$ II galaxies of the second data emission 
release of the SDSSI reobserved with the $4.2 \mathrm{~m}$ William Herschel Telescope (WHT), finding $t^{2}$ 's values close to zero for two of the objects, and a relatively large value $\left(t^{2} \sim 0.066 \pm 0.026\right)$ for the third. On the other hand, Guseva et al. (2006, 2007) have made a detailed study of a sample of 47 low metallicity H II regions in blue compact dwarf (BCD) galaxies and and 69 emission-line galaxies from the data release 3 of the SDSS and M101. These authors have measured the Balmer and Paschen jumps with the purpose of determining $T_{\mathrm{e}}(\mathrm{H} \mathrm{I})$. From Monte Carlo simulations, they fit the spectral energy distribution (SED) of the galaxies and find that the temperatures in the $\mathrm{O}^{++}$zone -derived from [O III] $\lambda \lambda(49595007) / \lambda 4363$ ratio- are, considering the dispersion of the data, equal to the temperature of the $\mathrm{H}^{+}$zone, obtained from fitting the Balmer jump and the SED. In any case, the dispersion of the data does not allow to discard small temperature fluctuations of about $\sim 3 \%-5 \%$.

Tsamis et al. (2003) proposed a chemically inhomogeneous scenario for H II regions similar to that proposed for PNe, but only very recently a physically reasonable model has been drawn (Tsamis \& Péquignot 2005; Stasińska et al. 2007). This scenario implies the presence of temperature fluctuations in the nebulae, but its resulting abundance pattern is not identical to the one found under the standard temperature fluctuations paradigm (see Stasińska et al. 2007). Those authors postulate the presence of a gas component of high metallicity, and consequently low $T_{\mathrm{e}}$ embedded in a less dense ambient gas, with lower metallicity and therefore larger temperature. According to Tsamis \& Péquignot (2005) and Stasińska et al. (2007), this denser component could come from the resulting material of type II SNe that has not been mixed with the bulk of ISM and that is in pressure balance with the normal composition ambient gas (an scenario originally proposed by Tenorio-Tagle 1996). According to this model, these inclusions would be responsible for most the ORLs emission and they would not emit in CELs due to their low temperature.

In this paper, we present a global analysis of some results of our study of a sample of H II regions mainly focussed on discussing the properties and behavior of the abundance discrepancy problem in these objects.

The structure of this paper is as follows: in $\S 2$ we briefly describe the data we have analized. In $\S 3$ we explore the behavior of the abundance discrepancy factor (ADF) with respect to some nebular parameters. In $\S$ we present some observational arguments against the two phase model in H II regions. Finally in $\S 5$ we briefly draw the conclusions of this work.

\footnotetext{
${ }^{2}$ Sloan Digital Sky Survey
} 


\section{The data}

The observations were made in two runs: March 2002 and March 2003 with the Ultraviolet Visual Echelle Spectrograph (UVES) at the Very Large Telescope (VLT) Kueyen unit in Cerro Paranal Observatory (Chile). A detailed description of the instrument and telescope configuration, as well as of data reduction and analysis, can be found in previous papers (Esteban et al. 2004; García-Rojas et al. 2004, 2005, 2006, 2007; López-Sánchez et al. 2007).

With the aim of presenting a homogeneous data set, we have recomputed the physical conditions and ionic and total abundances of some of our published results for some objects (NGC 3576, Orion nebula, NGC 3603) using the same atomic data and ionization correction factors (ICF) scheme than for the rest of the sample nebulae. Also, we have corrected some misprints ocurring in previous papers. In Table 1, we present the physical conditions of the ionized gas computed for our sample; it can be noted that only small corrections in the adopted $T_{\mathrm{e}}$ and $n_{\mathrm{e}}$ have been done with respect to the previously published ones. In Table 2, we present the estimated $t^{2}$ values, which are essentially the same as the previously published ones. Also, in Table 3 we present the compilation of the ionic abundances from ORLs. In Tables 1 and 5, we present the recomputed ionic and total abundances from CELs. We have re-calculated the electron density for the Orion nebula, NGC 3576 and NGC 3603. These new densities imply new values for the ionic abundances for the most density dependent ionic abundances i. e. $\mathrm{O}^{+}, \mathrm{S}^{+}, \mathrm{Cl}^{++}, \mathrm{Fe}^{3+}$ and $\mathrm{Ar}^{+3}$. Changes in the adopted atomic data for $\mathrm{O}^{+}$and $\mathrm{S}^{++}$(see García-Rojas et al. 2005) have affected the determination of the ionic abundances and physical conditions of NGC 3576 and the Orion nebula. However, the combined effect of these corrections is small for NGC 3576 and NGC 3603 (less than 0.03 dex). For the Orion nebula the effect is somewhat more important, reaching a factor of about 0.1 dex for $\mathrm{O}^{+}$.

Finally, we have re-computed He abundances for all the objects adopting a new set of ICFs: for the low ionization objects we have adopted the ICF by Peimbert \& Torres-Peimbert (1977), whereas for the high ionization ones we have adopted the ICF by Peimbert et al. (1992).

\section{Behavior of the ADF with respect to some nebular parameters.}

The different hypothesis proposed to explain the abundance discrepancy predict the existence or absence of correlations between the ADF and some nebular properties. Although these correlations have been exhaustively explored for PNe, there has not been a systematic study for H II regions. The aim of this section is to analyze possible correlations between the 
Table 1. Plasma diagnostics.

\begin{tabular}{|c|c|c|c|c|c|}
\hline Diagnostic & Line & M16 & M8 & M17 & M20 \\
\hline \multirow[t]{7}{*}{$n_{\mathrm{e}}\left(\mathrm{cm}^{-3}\right)$} & {$[\mathrm{NI}](\lambda 5198) /(\lambda 5200)$} & $1100_{-400}^{+750}$ & $1600_{-470}^{+750}$ & $1200_{-500}^{+1250}$ & $560_{-220}^{+340}$ \\
\hline & {$[\mathrm{O} \mathrm{II}](\lambda 3726) /(\lambda 3729)$} & $1050 \pm 250$ & $1800 \pm 800$ & $480 \pm 150$ & $240 \pm 70$ \\
\hline & {$[\mathrm{S} \mathrm{II}](\lambda 6716) /(\lambda 6731)$} & $1390 \pm 550$ & $1600 \pm 450$ & $500 \pm 220$ & $320 \pm 130$ \\
\hline & {$[\mathrm{Fe} \mathrm{III}]$} & $540_{-500}^{+>1000}$ & $2600 \pm 1450$ & $430_{-400}^{+>1000}$ & $560 \pm 390$ \\
\hline & {$[\mathrm{Cl} \mathrm{III]}(\lambda 5518) /(\lambda 5538)$} & $1370 \pm 1000$ & $2100 \pm 700$ & $270_{-270}^{+630}$ & $350_{-350}^{+780}$ \\
\hline & {$[\mathrm{Ar} I \mathrm{IV}](\lambda 4711) /(\lambda 4740)$} & $\ldots$ & $2450:^{\mathrm{d}}$ & $>800$ & $\ldots$ \\
\hline & $n_{\mathrm{e}}($ adopted $)$ & $1120 \pm 220$ & $1800 \pm 350$ & $470 \pm 120$ & $270 \pm 60$ \\
\hline \multirow[t]{13}{*}{$T_{\mathrm{e}}(\mathrm{K})$} & {$[\mathrm{N} \mathrm{II}](\lambda 6548+\lambda 6583) /(\lambda 5755)^{\mathrm{a}}$} & $8450 \pm 270$ & $8470 \pm 180$ & $8950 \pm 380$ & $8500 \pm 240$ \\
\hline & {$[\mathrm{S} \mathrm{II}](\lambda 6716+\lambda 6731) /(\lambda 4069+\lambda 4076)$} & $7520 \pm 430$ & $7220 \pm 300$ & $7100 \pm 750$ & $6950 \pm 350$ \\
\hline & {$[\mathrm{O}$ II $](\lambda 3726+\lambda 3729) /(\lambda 7320+\lambda 7330)^{\mathrm{a}}$} & $8260 \pm 400$ & $8700 \pm 350$ & $8750 \pm 550$ & $8275 \pm 400$ \\
\hline & $T_{\mathrm{e}}($ Low $)$ & $8350 \pm 200$ & $8500 \pm 150$ & $8870 \pm 300$ & $8400 \pm 200$ \\
\hline & {$[\mathrm{O}$ III $](\lambda 4959+\lambda 5007) /(\lambda 4363)$} & $7650 \pm 250$ & $8090 \pm 140$ & $8020 \pm 170$ & $7800 \pm 300$ \\
\hline & {$[$ Ar III $](\lambda 7136+\lambda 7751) /(\lambda 5192)$} & $\ldots$ & $7550 \pm 420$ & $8380 \pm 570$ & $8730 \pm 920^{\mathrm{b}}$ \\
\hline & {$[\mathrm{S} \mathrm{III}](\lambda 9069+\lambda 9532) /(\lambda 6312)$} & $8430 \pm 450$ & $8600 \pm 300^{\mathrm{c}}$ & $8110 \pm 400$ & $8300 \pm 400$ \\
\hline & $T_{\mathrm{e}}(\mathrm{High})$ & $7850 \pm 220$ & $8150 \pm 120$ & $8050 \pm 150$ & $7980 \pm 250$ \\
\hline & $T_{\mathrm{e}}(\mathrm{O} \mathrm{II}+\mathrm{III})$ & $8180 \pm 300$ & $8570 \pm 200$ & $8200 \pm 200$ & $8230 \pm 350$ \\
\hline & $\mathrm{He}$ II & $7300 \pm 350$ & $7500 \pm 200$ & $7450 \pm 200$ & $7650 \pm 300$ \\
\hline & Balmer discontinuity & $5450 \pm 820$ & $7100 \pm 1100$ & $\cdots$ & $6000 \pm 1300$ \\
\hline & Paschen discontinuity & $7200 \pm 1300$ & $7750 \pm 900$ & $6500 \pm 1000$ & $5700 \pm 1300$ \\
\hline & & NGC 3576 & ORION & NGC 3603 & S 311 \\
\hline \multirow[t]{7}{*}{$n_{\mathrm{e}}\left(\mathrm{cm}^{-3}\right)$} & {$[\mathrm{N} \mathrm{I}](\lambda 5198) /(\lambda 5200)$} & $\cdots$ & $1700 \pm 600$ & $4000:^{\mathrm{d}}$ & $590_{-200}^{+260}$ \\
\hline & {$[\mathrm{O} \mathrm{II}](\lambda 3726) /(\lambda 3729)$} & $1900 \pm 400$ & $6300_{-1600}^{+2800}$ & $3000 \pm 1000$ & $260 \pm 110$ \\
\hline & {$[\mathrm{S} \mathrm{II}](\lambda 6716) /(\lambda 6731)$} & $1300_{-300}^{+500}$ & $6500_{-1200}^{+2000}$ & $4150_{-1650}^{+3350}$ & $360_{-120}^{+140}$ \\
\hline & {$[\mathrm{Fe}$ III $]$} & $3100 \pm 1300$ & $9300 \pm 2700$ & $1330:^{-1050}$ & $390 \pm 220$ \\
\hline & {$[\mathrm{Cl}$ III $](\lambda 5518) /(\lambda 5538)$} & $3500_{-700}^{+900}$ & $9400_{-700}^{+1200}$ & $5600_{-2400}^{+3900}$ & $550_{-550}^{+650}$ \\
\hline & {$[\mathrm{Ar} I V](\lambda 4711) /(\lambda 4740)$} & $4500_{-1800}^{+2600}$ & $6800_{-1000}^{+1100}$ & $\leq 4000$ & $\ldots^{-550}$ \\
\hline & $n_{\mathrm{e}}($ adopted $)$ & $2300 \pm 300$ & $7800 \pm 600$ & $3400 \pm 850$ & $310 \pm 80$ \\
\hline \multirow[t]{12}{*}{$T_{\mathrm{e}}(\mathrm{K})$} & {$[\mathrm{N} \mathrm{II}](\lambda 6548+\lambda 6583) /(\lambda 5755)^{\mathrm{a}}$} & $8500 \pm 200$ & $10150 \pm 350$ & $11050 \pm 800$ & $9500 \pm 250$ \\
\hline & {$[\mathrm{S} \mathrm{II}](\lambda 6716+\lambda 6731) /(\lambda 4069+\lambda 4076)$} & $8400_{-600}^{+350}$ & $9050 \pm 800$ & $11050_{-2050}^{+3550}$ & $7200_{-600}^{+750}$ \\
\hline & {$[\mathrm{O} \mathrm{II}](\lambda 3726+\lambda 3729) /(\lambda 7320+\lambda 7330)^{\mathrm{a}}$} & $8050 \pm 450$ & $8700 \pm 500$ & $12350 \pm 1250$ & $9800 \pm 600$ \\
\hline & $T_{\mathrm{e}}($ Low $)$ & $8400 \pm 200$ & $9600 \pm 300$ & $11400 \pm 700$ & $9550 \pm 250$ \\
\hline & {$[\mathrm{O}$ III $](\lambda 4959+\lambda 5007) /(\lambda 4363)$} & $8500 \pm 50$ & $8300 \pm 40$ & $9060 \pm 200$ & $9000 \pm 200$ \\
\hline & {$[$ Ar III $](\lambda 7136+\lambda 7751) /(\lambda 5192)$} & $8600_{-350}^{+450}$ & $8300 \pm 400$ & $\ldots$ & $8800_{-850}^{+700}$ \\
\hline & {$[\mathrm{S} \mathrm{III}](\lambda 9069+\lambda 9532) /(\lambda 6312)$} & $8750 \pm 550$ & $9700_{-1200}^{+800}$ & $8800 \pm 500^{\mathrm{c}}$ & $9300 \pm 350^{\mathrm{c}}$ \\
\hline & $T_{\mathrm{e}}(\mathrm{High})$ & $8500 \pm 50$ & $8320 \pm 40$ & $9030 \pm 200$ & $9050 \pm 200$ \\
\hline & $T_{\mathrm{e}}(\mathrm{O} \mathrm{II}+\mathrm{III})$ & $8500 \pm 50$ & $8430 \pm 130$ & $9600 \pm 200$ & $9600 \pm 450$ \\
\hline & He II & $6800 \pm 400$ & $7950 \pm 200$ & $8480 \pm 200$ & $8750 \pm 500$ \\
\hline & Balmer discontinuity & $6650 \pm 750$ & $7900 \pm 600$ & $\cdots$ & $9500 \pm 900$ \\
\hline & Paschen discontinuity & $6700 \pm 900$ & $8100 \pm 1400$ & $6900 \pm 1100$ & $8700 \pm 1100$ \\
\hline
\end{tabular}

${ }^{\mathrm{a}}$ The recombination contribution to the auroral lines has been taken into account.

b The [Ar III] $\lambda 7751$ line is severely blended with a telluric line.

c [S III] $\lambda 9069$ or $\lambda 9530$ affected by atmospheric absorption bands.

${ }^{\mathrm{d} C o l o n s}$ indicate very high uncertainties. These values has not been taken into account in the adopted average. 
Table 2. $t^{2}$ parameter.

\begin{tabular}{lcccc}
\hline \hline \multicolumn{1}{c}{ Method } & M16 & M 8 & M17 & M20 \\
\hline $\mathrm{O}^{+}(\mathrm{R} / \mathrm{C})$ & $\ldots$ & $0.031 \pm 0.017$ & $0.109:$ & $0.032 \pm 0.020$ \\
$\mathrm{O}^{++}(\mathrm{R} / \mathrm{C})$ & $0.046 \pm 0.007$ & $0.045 \pm 0.005$ & $0.034 \pm 0.005$ & $0.038 \pm 0.016$ \\
$\mathrm{C}^{++}(\mathrm{R} / \mathrm{C})$ & $\ldots$ & $0.035 \pm 0.005$ & $\ldots$ & $\ldots$ \\
$\mathrm{Ne}^{++}(\mathrm{R} / \mathrm{C})$ & $\ldots$ & $\ldots$ & $\ldots$ & $\ldots$ \\
$\mathrm{He}^{+}$ & $0.017 \pm 0.013$ & $0.046 \pm 0.009$ & $0.027 \pm 0.014$ & $0.017 \pm 0.010$ \\
$\mathrm{Bac} / \mathrm{Pac}-\mathrm{LEC}$ & $0.045 \pm 0.014$ & $0.022 \pm 0.015$ & $0.035 \pm 0.021$ & $0.049 \pm 0.019$ \\
$\mathrm{Adopted}$ & $\mathbf{0 . 0 3 9} \pm \mathbf{0 . 0 0 6}$ & $\mathbf{0 . 0 4 0} \pm \mathbf{0 . 0 0 4}$ & $\mathbf{0 . 0 3 3} \pm \mathbf{0 . 0 0 5}$ & $\mathbf{0 . 0 2 9} \pm \mathbf{0 . 0 0 7}$ \\
\hline \multicolumn{1}{c}{ Method } & $\mathrm{NGC} 3576$ & $\mathrm{ORION}$ & $\mathrm{NGC} 3603$ & $\mathrm{~S} 311$ \\
\hline $\mathrm{O}^{+}(\mathrm{R} / \mathrm{C})$ & $\ldots$ & $0.063 \pm 0.029$ & $\ldots$ & $\ldots$ \\
$\mathrm{O}^{++}(\mathrm{R} / \mathrm{C})$ & $0.038 \pm 0.006$ & $0.020 \pm 0.002$ & $0.042 \pm 0.009$ & $0.040 \pm 0.008$ \\
$\mathrm{C}^{++}(\mathrm{R} / \mathrm{C})$ & $\ldots$ & $0.039 \pm 0.011$ & $\ldots$ & $\ldots$ \\
$\mathrm{Ne} \mathrm{N}^{++}(\mathrm{R} / \mathrm{C})$ & $0.036_{-0.024}^{+0.014}$ & $0.034 \pm 0.014$ & $\ldots$ & $\ldots$ \\
$\mathrm{He}$ & $0.023 \pm 0.019$ & $0.022 \pm 0.002$ & $0.032 \pm 0.014$ & $0.034 \pm 0.010$ \\
$\mathrm{Bac} / \mathrm{Pac}-\mathrm{LEC}$ & $0.037 \pm 0.012$ & $0.016 \pm 0.014$ & $0.056 \pm 0.023$ & $0.009:$ \\
$\mathrm{Adopted}$ & $\mathbf{0 . 0 3 8} \pm \mathbf{0 . 0 0 9}$ & $\mathbf{0 . 0 2 2} \pm \mathbf{0 . 0 0 2}$ & $\mathbf{0 . 0 4 0} \pm \mathbf{0 . 0 0 8}$ & $\mathbf{0 . 0 3 8} \pm \mathbf{0 . 0 0 7}$ \\
\hline
\end{tabular}

Table 3. Heavy element ionic abundances from ORLs.

\begin{tabular}{ccccc}
\hline \hline \multicolumn{5}{c}{$12+\log \left(\mathrm{X}^{+i} / \mathrm{H}^{+}\right)$} \\
\hline Ion & $\mathrm{M} 16$ & $\mathrm{M} 8$ & $\mathrm{M} 17$ & $\mathrm{M} 20$ \\
\hline $\mathrm{O}^{+}$ & $\ldots$ & $8.53 \pm 0.06$ & $\ldots$ & $8.62 \pm 0.07$ \\
$\mathrm{O}^{++}$ & $8.30 \pm 0.04$ & $8.23 \pm 0.02$ & $8.68 \pm 0.02$ & $8.00 \pm 0.18$ \\
$\mathrm{C}^{++}$ & $8.40 \pm 0.03$ & $8.30 \pm 0.02$ & $8.68 \pm 0.03$ & $8.18 \pm 0.05$ \\
$\mathrm{Ne}^{++}$ & $\ldots$ & $\ldots$ & $\ldots$ & $\ldots$ \\
\hline Ion & NGC 3576 & ORION & NGC 3603 & $\mathrm{~S} \mathrm{311}$ \\
\hline $\mathrm{O}^{+}$ & $\ldots$ & $\ldots$ & $\ldots$ & $\ldots$ \\
$\mathrm{O}^{++}$ & $8.62 \pm 0.05$ & $8.57 \pm 0.01$ & $8.71 \pm 0.05$ & $8.08 \pm 0.03$ \\
$\mathrm{C}^{++}$ & $8.45 \pm 0.06$ & $8.34 \pm 0.02$ & $8.48 \pm 0.07$ & $8.00 \pm 0.04$ \\
$\mathrm{Ne}^{++}$ & $7.90 \pm 0.14$ & $7.95 \pm 0.09$ & $\cdots$ & $\cdots$ \\
\hline
\end{tabular}


Table 4. Heavy element ionic abundances from CELs.

\begin{tabular}{|c|c|c|c|c|c|c|c|c|}
\hline \multirow[b]{3}{*}{ Ion } & \multicolumn{8}{|c|}{$12+\log \left(\mathrm{X}^{+i} / \mathrm{H}^{+}\right)$} \\
\hline & \multicolumn{2}{|c|}{ M16 } & \multicolumn{2}{|c|}{ M8 } & \multicolumn{2}{|c|}{ M17 } & \multicolumn{2}{|c|}{ M20 } \\
\hline & $t^{2}=0.000$ & $t^{2}=0.039$ & $t^{2}=0.000$ & $t^{2}=0.040$ & $t^{2}=0.000$ & $t^{2}=0.033$ & $t^{2}=0.000$ & $t^{2}=0.029$ \\
\hline $\mathrm{N}^{0}$ & $6.15 \pm 0.06$ & $6.33 \pm 0.07$ & $5.81 \pm 0.05$ & $5.99 \pm 0.06$ & $5.57 \pm 0.07$ & $5.70 \pm 0.07$ & $5.90 \pm 0.07$ & $6.03 \pm 0.08$ \\
\hline $\mathrm{N}^{+}$ & $7.71 \pm 0.05$ & $7.88 \pm 0.06$ & $7.50 \pm 0.03$ & $7.67 \pm 0.04$ & $6.82 \pm 0.10$ & $6.94 \pm 0.10$ & $7.55 \pm 0.04$ & $7.67 \pm 0.05$ \\
\hline $\mathrm{O}^{0}$ & $7.23 \pm 0.05$ & $7.40 \pm 0.06$ & $6.40 \pm 0.03$ & $6.57 \pm 0.04$ & $6.87 \pm 0.07$ & $7.00 \pm 0.07$ & $6.60 \pm 0.05$ & $6.72 \pm 0.06$ \\
\hline $\mathrm{O}^{+}$ & $8.47 \pm 0.08$ & $8.66 \pm 0.09$ & $8.39 \pm 0.06$ & $8.58 \pm 0.07$ & $7.84 \pm 0.09$ & $7.98 \pm 0.09$ & $8.46 \pm 0.07$ & $8.59 \pm 0.08$ \\
\hline $\mathrm{O}^{++}$ & $7.85 \pm 0.07$ & $8.18 \pm 0.10$ & $7.86 \pm 0.03$ & $8.18 \pm 0.07$ & $8.41 \pm 0.04$ & $8.67 \pm 0.06$ & $7.67 \pm 0.08$ & $7.90 \pm 0.10$ \\
\hline $\mathrm{Ne}^{++}$ & $7.01 \pm 0.07$ & $7.38 \pm 0.10$ & $6.95 \pm 0.05$ & $7.30 \pm 0.07$ & $7.64 \pm 0.04$ & $7.93 \pm 0.07$ & $6.55 \pm 0.09$ & $6.80 \pm 0.11$ \\
\hline $\mathrm{S}^{+}$ & $6.32 \pm 0.05$ & $6.49 \pm 0.06$ & $5.93 \pm 0.04$ & $6.10 \pm 0.07$ & $5.44 \pm 0.05$ & $5.56 \pm 0.06$ & $6.17 \pm 0.05$ & $6.29 \pm 0.06$ \\
\hline $\mathrm{S}^{++}$ & $6.84 \pm 0.06$ & $7.22 \pm 0.10$ & $6.89 \pm 0.03$ & $7.25 \pm 0.07$ & $6.90 \pm 0.04$ & $7.19 \pm 0.06$ & $6.79 \pm 0.06$ & $7.09 \pm 0.10$ \\
\hline $\mathrm{Cl}^{+}$ & $4.77 \pm 0.05$ & $4.91 \pm 0.07$ & $4.53 \pm 0.04$ & $4.66 \pm 0.06$ & $3.95_{-0.12}^{+0.09}$ & $4.06_{-0.12}^{+0.09}$ & $4.75 \pm 0.05$ & $4.85 \pm 0.07$ \\
\hline $\mathrm{Cl}^{++}$ & $5.04 \pm 0.06$ & $5.36 \pm 0.08$ & $5.02 \pm 0.04$ & $5.32 \pm 0.06$ & $5.04 \pm 0.04$ & $5.29 \pm 0.06$ & $4.99 \pm 0.07$ & $5.21 \pm 0.08$ \\
\hline $\mathrm{Cl}^{3+}$ & $\ldots$ & $\ldots$ & $\ldots$ & $\ldots$ & $3.15:$ & 3.35: & $\ldots$ & $\ldots$ \\
\hline $\mathrm{Ar}^{++}$ & $6.25 \pm 0.05$ & $6.53 \pm 0.08$ & $6.21 \pm 0.03$ & $6.48 \pm 0.05$ & $6.35 \pm 0.04$ & $6.57 \pm 0.06$ & $6.17 \pm 0.06$ & $6.36 \pm 0.08$ \\
\hline $\mathrm{Ar}^{3+}$ & $3.89 \pm 0.22$ & $4.23 \pm 0.23$ & $3.69 \pm 0.09$ & $4.01 \pm 0.10$ & $4.15_{-0.18}^{+0.12}$ & $4.42_{-0.18}^{+0.13}$ & $4.01 \pm 0.18$ & $4.24 \pm 0.19$ \\
\hline $\mathrm{Fe}^{+}$ & 4.62: & 4.78: & 4.61: & 4.77: & 4.05: & 4.17: & 4.51: & 4.62: \\
\hline $\mathrm{Fe}^{++}$ & $5.07 \pm 0.04$ & $5.41 \pm 0.08$ & $5.58 \pm 0.04$ & $5.91 \pm 0.06$ & $5.24 \pm 0.06$ & $5.51 \pm 0.08$ & $5.23 \pm 0.10$ & $5.47 \pm 0.12$ \\
\hline \multirow[t]{3}{*}{$\mathrm{Fe}^{3+}$} & $\ldots$ & $\ldots$ & $\ldots$ & $\ldots$ & $\ldots$ & & $\ldots$ & $\ldots$ \\
\hline & \multicolumn{8}{|c|}{$12+\log \left(\mathrm{X}^{+i} / \mathrm{H}^{+}\right)$} \\
\hline & \multicolumn{2}{|c|}{ NGC 3576} & \multicolumn{2}{|c|}{ ORION } & \multicolumn{2}{|c|}{ NGC 3603} & \multicolumn{2}{|c|}{ S 311} \\
\hline Ion & $t^{2}=0.000$ & $t^{2}=0.038$ & $t^{2}=0.000$ & $t^{2}=0.022$ & $t^{2}=0.000$ & $t^{2}=0.040$ & $t^{2}=0.000$ & $t^{2}=0.038$ \\
\hline $\mathrm{N}^{0}$ & $\ldots$ & $\ldots$ & $5.69 \pm 0.06$ & $5.76 \pm 0.06$ & $5.55 \pm 0.11$ & $5.65 \pm 0.11$ & $5.74 \pm 0.06$ & $5.88 \pm 0.07$ \\
\hline $\mathrm{N}^{+}$ & $7.09 \pm 0.06$ & $7.25 \pm 0.07$ & $6.97 \pm 0.09$ & $7.03 \pm 0.09$ & $6.45 \pm 0.07$ & $6.55 \pm 0.07$ & $7.25 \pm 0.05$ & $7.38 \pm 0.06$ \\
\hline $\mathrm{O}^{0}$ & $6.35 \pm 0.04$ & $6.51 \pm 0.06$ & $6.22 \pm 0.05$ & $6.28 \pm 0.05$ & $6.32 \pm 0.09$ & $6.42 \pm 0.09$ & $6.74 \pm 0.06$ & $6.87 \pm 0.06$ \\
\hline $\mathrm{O}^{+}$ & $8.21 \pm 0.07$ & $8.38 \pm 0.08$ & $7.93 \pm 0.15$ & $8.00 \pm 0.15$ & $7.42 \pm 0.11$ & $7.52 \pm 0.11$ & $8.26 \pm 0.07$ & $8.40 \pm 0.08$ \\
\hline $\mathrm{O}^{++}$ & $8.35 \pm 0.03$ & $8.63 \pm 0.08$ & $8.42 \pm 0.01$ & $8.58 \pm 0.03$ & $8.42 \pm 0.05$ & $8.68 \pm 0.08$ & $7.81 \pm 0.04$ & $8.05 \pm 0.06$ \\
\hline $\mathrm{Ne}^{++}$ & $7.61 \pm 0.09$ & $7.91 \pm 0.12$ & $7.67 \pm 0.07$ & $7.84 \pm 0.07$ & $7.72 \pm 0.05$ & $8.00 \pm 0.08$ & $6.81 \pm 0.05$ & $7.07 \pm 0.07$ \\
\hline $\mathrm{S}^{+}$ & $5.75 \pm 0.08$ & $5.91 \pm 0.09$ & $5.44 \pm 0.06$ & $5.50 \pm 0.06$ & $5.08 \pm 0.10$ & $5.17 \pm 0.10$ & $6.03 \pm 0.05$ & $6.15 \pm 0.06$ \\
\hline $\mathrm{S}^{++}$ & $6.86 \pm 0.10$ & $7.17 \pm 0.10$ & $6.95 \pm 0.04$ & $7.12 \pm 0.05$ & $6.83 \pm 0.04$ & $7.11 \pm 0.09$ & $6.68 \pm 0.07$ & $6.95 \pm 0.09$ \\
\hline $\mathrm{Cl}^{+}$ & $4.13 \pm 0.08$ & $4.26 \pm 0.08$ & $3.80 \pm 0.11^{\mathrm{a}}$ & $3.85 \pm 0.11^{\mathrm{a}}$ & $3.46 \pm 0.07$ & $3.54 \pm 0.07$ & $4.56 \pm 0.05$ & $4.67 \pm 0.05$ \\
\hline $\mathrm{Cl}^{++}$ & $4.95 \pm 0.06$ & $5.21_{-0.08}^{+0.10}$ & $5.13 \pm 0.02$ & $5.28 \pm 0.02$ & $5.07 \pm 0.05$ & $5.31 \pm 0.08$ & $4.85 \pm 0.05$ & $5.08 \pm 0.05$ \\
\hline $\mathrm{Cl}^{3+}$ & $3.21 \pm 0.07$ & $3.42 \pm 0.09$ & $3.66 \pm 0.12$ & $3.78 \pm 0.12$ & $3.86 \pm 0.04$ & $4.06 \pm 0.07$ & $\ldots$ & $\ldots$ \\
\hline $\mathrm{Ar}^{++}$ & $6.34 \pm 0.05$ & $6.57 \pm 0.08$ & $6.37 \pm 0.05$ & $6.50 \pm 0.05$ & $6.35 \pm 0.04$ & $6.56 \pm 0.07$ & $6.08 \pm 0.04$ & $6.28 \pm 0.06$ \\
\hline $\mathrm{Ar}^{3+}$ & $4.20 \pm 0.07$ & $4.48 \pm 0.10$ & $4.59 \pm 0.03$ & $4.75 \pm 0.04$ & $4.85 \pm 0.06$ & $5.11 \pm 0.08$ & $3.42_{-0.30}^{+0.18}$ & $3.66_{-0.30}^{+0.18}$ \\
\hline $\mathrm{Fe}^{+}$ & 4.61: & 4.76: & 4.54: & 4.60: & 4.04: & 4.13: & 4.25: & 4.37: \\
\hline $\mathrm{Fe}^{++}$ & $5.57 \pm 0.05$ & $5.85 \pm 0.09$ & $5.43 \pm 0.06$ & $5.59 \pm 0.06$ & $5.24 \pm 0.06$ & $5.50 \pm 0.09$ & $5.05 \pm 0.06$ & $5.30 \pm 0.08$ \\
\hline $\mathrm{Fe}^{3+}$ & $5.71_{-0.29}^{+0.17}$ & $5.95_{-0.29}^{+0.17}$ & $5.65_{-0.30}^{+0.19}$ & $5.78_{-0.30}^{+0.19}$ & $\ldots$ & $\ldots$ & $\ldots$ & $\ldots$ \\
\hline
\end{tabular}

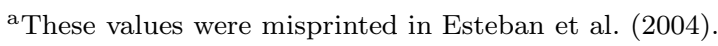


Table 5. Total abundances.

\begin{tabular}{|c|c|c|c|c|c|c|c|c|}
\hline \multicolumn{9}{|c|}{$12+\log \left(\mathrm{X}^{+i} / \mathrm{H}^{+}\right)$} \\
\hline & \multicolumn{2}{|c|}{ M16 } & \multicolumn{2}{|c|}{ M8 } & \multicolumn{2}{|c|}{ M17 } & \multicolumn{2}{|c|}{ M20 } \\
\hline & $t^{2}=0.000$ & $t^{2}=0.039$ & $t^{2}=0.000$ & $t^{2}=0.040$ & $t^{2}=0.000$ & $t^{2}=0.033$ & $t^{2}=0.000$ & $t^{2}=0.029$ \\
\hline $\mathrm{He}$ & $11.01 \pm 0.02$ & $10.97 \pm 0.02$ & $10.87 \pm 0.01$ & $10.85 \pm 0.01$ & $10.97 \pm 0.01$ & $10.97 \pm 0.01$ & $10.95 \pm 0.06$ & $10.92 \pm 0.06$ \\
\hline $\mathrm{C}^{\mathrm{a}}$ & $8.85 \pm 0.10$ & $8.85 \pm 0.10$ & $8.61 / 8.69 \pm 0.09$ & $8.69 / 8.69 \pm 0.09$ & $8.77 \pm 0.04$ & $8.77 \pm 0.04$ & $8.66 \pm 0.11$ & $8.66 \pm 0.11$ \\
\hline $\mathrm{N}$ & $7.84 \pm 0.06$ & $8.07 \pm 0.12$ & $7.72 \pm 0.03$ & $7.96 \pm 0.06$ & $7.62 \pm 0.12$ & $7.87 \pm 0.13$ & $7.67 \pm 0.05$ & $7.83 \pm 0.07$ \\
\hline $\mathrm{O}$ & $8.56 \pm 0.07$ & $8.78 \pm 0.07$ & $8.51 \pm 0.05$ & $8.73 \pm 0.05$ & $8.51 \pm 0.04$ & $8.75 \pm 0.05$ & $8.53 \pm 0.06$ & $8.67 \pm 0.07$ \\
\hline $\mathrm{O}^{\mathrm{b}}$ & $8.70 \pm 0.06$ & $8.70 \pm 0.06$ & $8.71 \pm 0.05$ & $8.71 \pm 0.05$ & $8.89 \pm 0.04$ & $8.89 \pm 0.04$ & $8.71 \pm 0.09$ & $8.71 \pm 0.09$ \\
\hline $\mathrm{O}^{\mathrm{c}}$ & $8.81 \pm 0.07$ & $8.81 \pm 0.07$ & $8.71 \pm 0.04$ & $8.71 \pm 0.04$ & $8.76 \pm 0.04$ & $8.76 \pm 0.04$ & $8.69 \pm 0.10$ & $8.69 \pm 0.10$ \\
\hline $\mathrm{Ne}^{\mathrm{d}}$ & $7.86 \pm 0.15$ & $8.08 \pm 0.17$ & $7.81 \pm 0.12$ & $8.03 \pm 0.13$ & $7.74 \pm 0.07$ & $8.01 \pm 0.09$ & $7.83 \pm 0.16$ & $7.97 \pm 0.18$ \\
\hline S & $6.96 \pm 0.05$ & $7.29 \pm 0.08$ & $6.94 \pm 0.03$ & $7.28 \pm 0.06$ & $7.01 \pm 0.04$ & $7.33 \pm 0.06$ & $6.88 \pm 0.05$ & $7.12 \pm 0.09$ \\
\hline $\mathrm{Cl}^{\mathrm{e}}$ & $5.23 \pm 0.04$ & $5.49 \pm 0.07$ & $5.14 \pm 0.04$ & $5.41 \pm 0.06$ & $5.08 \pm 0.04$ & $5.32 \pm 0.06$ & $5.19 \pm 0.05$ & $5.37 \pm 0.06$ \\
\hline $\mathrm{Ar}$ & $6.70 \pm 0.06$ & $6.84 \pm 0.08$ & $6.52 \pm 0.05$ & $6.69 \pm 0.06$ & $6.39 \pm 0.06$ & $6.59 \pm 0.07$ & $6.65 \pm 0.07$ & $6.70 \pm 0.09$ \\
\hline $\mathrm{Fe}^{\mathrm{f}}$ & $5.17 \pm 0.11$ & $5.53 \pm 0.13$ & $5.69 \pm 0.09$ & $6.04 \pm 0.10$ & $5.87 \pm 0.13$ & $6.22 \pm 0.15$ & $5.31 \pm 0.13$ & $5.56 \pm 0.15$ \\
\hline \multirow[t]{4}{*}{$\mathrm{Fe}^{\mathrm{g}}$} & $5.20 \pm 0.06$ & $5.51 \pm 0.07$ & $5.62 \pm 0.04$ & $5.94 \pm 0.06$ & $5.27 \pm 0.06$ & $5.52 \pm 0.08$ & $5.31 \pm 0.09$ & $5.52 \pm 0.10$ \\
\hline & \multicolumn{8}{|c|}{$12+\log \left(\mathrm{X}^{+i} / \mathrm{H}^{+}\right)$} \\
\hline & \multicolumn{2}{|c|}{ NGC 3576} & \multicolumn{2}{|c|}{ ORION } & \multicolumn{2}{|c|}{ NGC 3603} & \multicolumn{2}{|c|}{ S 311} \\
\hline & $t^{2}=0.000$ & $t^{2}=0.038$ & $t^{2}=0.000$ & $t^{2}=0.022$ & $t^{2}=0.000$ & $t^{2}=0.040$ & $t^{2}=0.000$ & $t^{2}=0.038$ \\
\hline $\mathrm{He}$ & $10.97 \pm 0.03$ & $10.96 \pm 0.04$ & $10.95 \pm 0.004$ & $10.95 \pm 0.004$ & $10.99 \pm 0.01$ & $10.99 \pm 0.01$ & $10.99 \pm 0.02$ & $10.97 \pm 0.02$ \\
\hline $\mathrm{C}^{\mathrm{a}}$ & $8.61 \pm 0.08$ & $8.61 \pm 0.08$ & $8.43 \pm 0.04$ & $8.43 \pm 0.04$ & $8.51 \pm 0.07$ & $8.51 \pm 0.07$ & $8.37 \pm 0.10$ & $8.37 \pm 0.10$ \\
\hline $\mathrm{N}$ & $7.57 \pm 0.06$ & $7.81 \pm 0.07$ & $7.73 \pm 0.09$ & $7.87 \pm 0.09$ & $7.62 \pm 0.13$ & $7.89 \pm 0.14$ & $7.43 \pm 0.06$ & $7.61 \pm 0.07$ \\
\hline $\mathrm{O}$ & $8.59 \pm 0.03$ & $8.82 \pm 0.07$ & $8.54 \pm 0.03$ & $8.68 \pm 0.04$ & $8.46 \pm 0.05$ & $8.71 \pm 0.07$ & $8.39 \pm 0.05$ & $8.56 \pm 0.06$ \\
\hline $\mathrm{O}^{\mathrm{b}}$ & $8.74 \pm 0.06$ & $8.74 \pm 0.06$ & $8.71 \pm 0.03$ & $8.71 \pm 0.03$ & $\ldots$ & $\ldots$ & $\ldots$ & $\ldots$ \\
\hline $\mathrm{O}^{\mathrm{c}}$ & $8.82 \pm 0.06$ & $8.82 \pm 0.06$ & $8.67 \pm 0.03$ & $8.67 \pm 0.03$ & $8.72 \pm 0.05$ & $8.72 \pm 0.05$ & $8.57 \pm 0.05$ & $8.57 \pm 0.05$ \\
\hline $\mathrm{Ne}^{\mathrm{d}}$ & $7.85 \pm 0.10$ & $8.11 \pm 0.12$ & $7.79 \pm 0.07$ & $7.94 \pm 0.07$ & $7.76 \pm 0.08$ & $8.03 \pm 0.11$ & $7.79 \pm 0.13$ & $7.98 \pm 0.14$ \\
\hline S & $6.92 \pm 0.10$ & $7.23 \pm 0.10$ & $7.04 \pm 0.04$ & $7.23 \pm 0.04$ & $7.04 \pm 0.05$ & $7.36 \pm 0.08$ & $6.77 \pm 0.06$ & $7.02 \pm 0.08$ \\
\hline $\mathrm{Cl}^{\mathrm{e}}$ & $5.02 \pm 0.05$ & $5.27 \pm 0.07$ & $5.14 \pm 0.04$ & $5.29 \pm 0.04$ & $5.11 \pm 0.05$ & $5.34 \pm 0.07$ & $5.03 \pm 0.06$ & $5.22 \pm 0.07$ \\
\hline $\mathrm{Ar}$ & $6.44 \pm 0.06$ & $6.65 \pm 0.09$ & $6.50 \pm 0.06$ & $6.63 \pm 0.06$ & $6.37 \pm 0.06$ & $6.58 \pm 0.08$ & $6.43 \pm 0.06$ & $6.56 \pm 0.07$ \\
\hline $\mathrm{Fe}^{\mathrm{f}}$ & $5.89 \pm 0.21$ & $6.23 \pm 0.10$ & $5.96 \pm 0.16$ & $6.18 \pm 0.14$ & $6.14 \pm 0.16$ & $6.53 \pm 0.19$ & $5.17 \pm 0.11$ & $5.44 \pm 0.13$ \\
\hline $\mathrm{Fe}^{\mathrm{g}}$ & $5.97_{-0.15}^{+0.11}$ & $6.22 \pm 0.12$ & $5.88 \pm 0.12$ & $6.02 \pm 0.12$ & $5.27 \pm 0.06$ & $5.52 \pm 0.09$ & $5.11 \pm 0.06$ & $5.35 \pm 0.08$ \\
\hline
\end{tabular}

${ }^{a}$ For M8 and Orion nebula, ICFs from C II] $\lambda 2525$ line/ICF from photoionization models by Garnett et al. (1999).

${ }^{\mathrm{b}} \mathrm{O}^{+} / \mathrm{H}^{+}$and $\mathrm{O}^{++} / \mathrm{H}^{+}$from $\mathrm{O}$ I and $\mathrm{O}$ II ORLs.

${ }^{\mathrm{c}} \mathrm{O}^{+} / \mathrm{H}^{+}$from $\left[\mathrm{O}\right.$ II] CELs and $t^{2}>0.00 . \mathrm{O}^{++} / \mathrm{H}^{+}$from O II ORLs.

${ }^{\mathrm{d}}$ ICF from Peimbert \& Costerd (1969) for M17, NGC 3576 and NGC 3603. ICF from Torres-Peimbert \& Peimbert 1977 for M16, M8, M20 and S 311.

${ }^{e} \mathrm{Cl} / \mathrm{H}=\mathrm{Cl}^{+} / \mathrm{H}^{+}+\mathrm{Cl}^{++} / \mathrm{H}^{+}$for M16, M8, M20, and S 311. $\mathrm{Cl} / \mathrm{H}=\mathrm{Cl}^{+} / \mathrm{H}^{+}+\mathrm{Cl}^{++} / \mathrm{H}^{+}+\mathrm{Cl}^{+3} / \mathrm{H}^{+}$for $\mathrm{M} 17, \mathrm{NGC} 3576$, Orion nebula and NGC 3603.

${ }^{\mathrm{f}} \mathrm{ICF}$ from Rodríguez \& Rubin (2005) equation (2).

${ }^{\mathrm{g}} \mathrm{Fe} / \mathrm{H}=\mathrm{Fe}^{+} / \mathrm{H}^{+}+\mathrm{Fe}^{++} / \mathrm{H}^{+}$for M16, M8, M17, M20, NGC 3603 and S 311. $\mathrm{Fe} / \mathrm{H}=\mathrm{Fe}^{+} / \mathrm{H}^{+}+\mathrm{Fe}^{++} / \mathrm{H}^{+}+\mathrm{Fe}^{+3} / \mathrm{H}^{+}$for NGC 3576 and Orion nebula. 
$\mathrm{ADF}$ and different nebular properties in H II regions (from our data and others available in the literature), with the purpose of discarding possible systematic errors and verifying the consistency of some hypothesis proposed to explain the abundance discrepancy.

Hereafter, the ADF found by Tsamis et al. (2003) for the SMC N11B H II region has been omitted in most of the figures and the discussion, because the ADF value for this region is much larger than for the rest of the H II regions and may be considerably overestimated. For this object, Tsamis et al. (2003) made an attempt to correct the intensity of the RLs of multiplet 1 of O II for the presence of stellar absorption features, mainly caused by B stars - that have a strong absorption O II spectra- in the area covered by the slit. In any case, this effect, which can be important in extragalactic objects, can only be corrected in the appropriate form if the spectral features of the stars are resolved, or if synthetic spectra are available, and this is not the case in this particular object. In fact, it is not possible to distinguish between the nebular emission and stellar absorption features in the spectra of Tsamis et al. 2003) of N11B. It is important to emphasize that this effect must be investigated whenever is possible to perform a suitable analysis when computing abundances from ORLs in extragalactic H II regions.

For all the fits, we have computed their Spearman's correlation coefficiens. These coefficients are the most appropriate ones for non gaussian distributions, as well as the two-sided significance of its deviation from zero; the significance is a value in the interval [0.0, 1.0], and a small value indicates a significant correlation.

One important result of our data compilation for $\mathrm{H}$ II regions is that the ADF is fairly constant for these objects and of the order of 2 . This is a crucial difference with the behavior shown by this factor in PNe, where it can vary from values of 1 up to 20 (see $\S 4.2$ for a further discussion and references).

In Figure 1 to 3 we have represented the ADF with respect to different nebular parameters, and in Table 6] we include the slope of the fits, their corresponding Spearman's coefficients, $r$, and the significance of the correlation, $P$, for each of the aforementioned diagrams. As it can be seen in Table the correlation coefficients for all the fits are very low and they have quite poor statistical significance. As in the rest of the cases, Figure 1 shows that the ADF seems to be not related to $\mathrm{O}^{++} / \mathrm{H}^{+}$nor $\mathrm{O}^{+} / \mathrm{O}^{++}$, at least within the observational uncertainties. The metallicity dependency of the $t^{2}$ parameter was suggested by Garnett (1992), who found that photoionization models could produce temperature fluctuations similar to the observed ones for nebulae with $T_{\mathrm{e}} \leq 9000 \mathrm{~K}$, increasing for colder (more metallic) nebulae. However, the observed $t^{2}$ does not seem to depend on metallicity, both for H II regions and $\mathrm{PNe}$. For $\mathrm{PNe}$, several authors have found that the large range of abundance discrepancies found might be mainly due to the fact that ADF increases monotonically with 


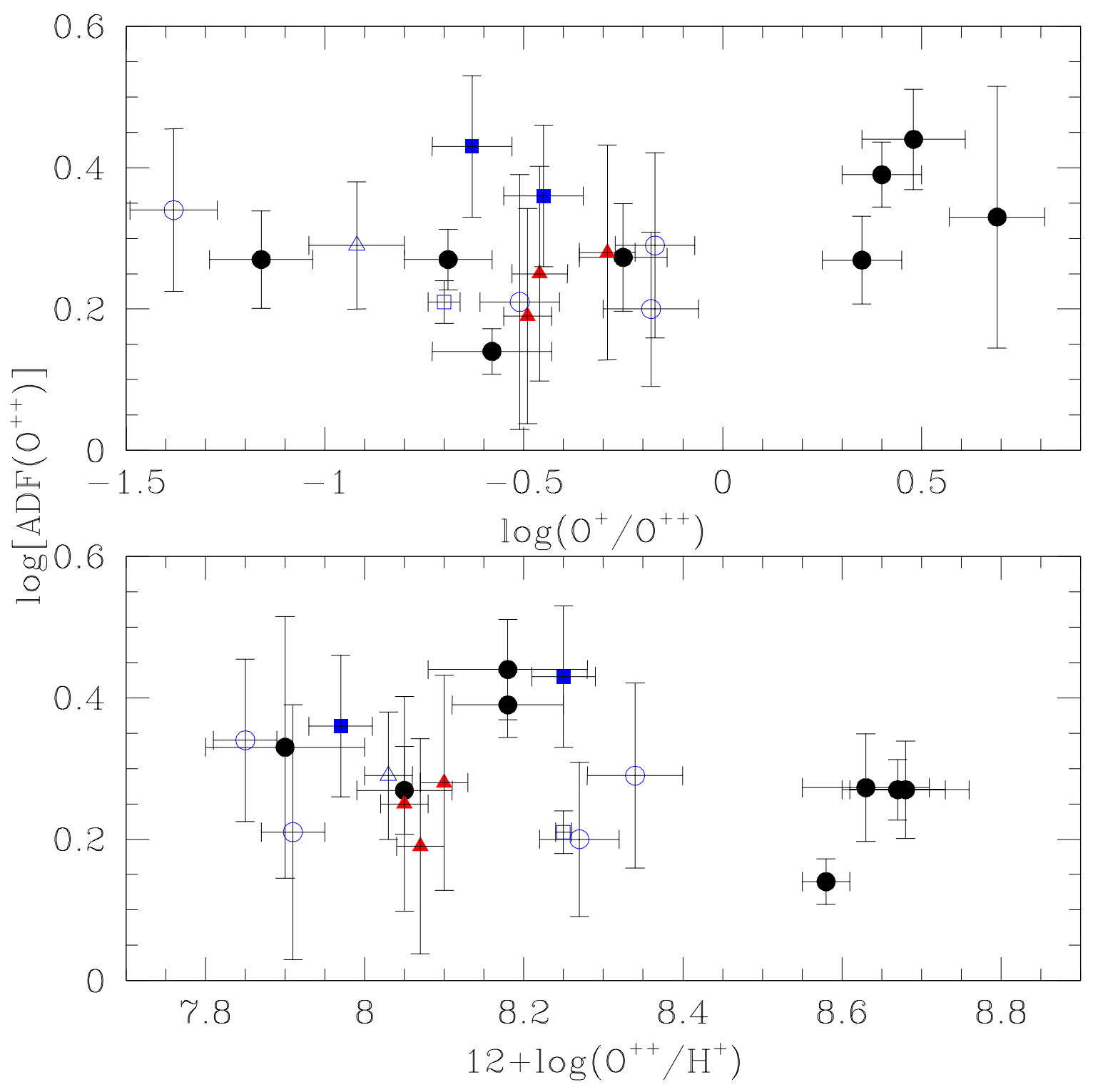

Fig. 1. $-\operatorname{ADF}\left(\mathrm{O}^{++}\right)$vs. the degree of ionization (upper panel) and $\mathrm{O}^{++} / \mathrm{H}^{+}$determined from CELs (lower panel). The Galactic H II regions of our sample are represented by filled dots; the rest of symbols represents data from the literature for extragalactic H II regions: filled triangles: López-Sánchez et al. (2007); filled squares: Tsamis et al. (2003); empty dots: Esteban et al. (2002); empty triangles: Peimbert \& Peimbert (2005); empty squares: Peimbert (2003). 
metallicity (see e.g. Liu et al. 2000, 2004). In the case of H II regions, as it can be observed in Figure 2, the ADF seems to remain relatively constant with the $\mathrm{O}$ abundance, determined either from CELs or ORLs, and within the uncertainties. We have also represented the ADF $v s$. the ratio between high and low ionization temperatures, finding that there is, again, no correlation within the observational errors (see Figure 3 and Table 6); this indicates that large scale variations of $T_{\mathrm{e}}$ due to natural temperature gradients throughout a nebula, do not seem to be related to the abundance discrepancy. As it can also been seen in Figure 3, we have verified that the ADF does not seem to depend on the assumed temperature, which discards systematic effects in the determination of abundances from CELs, at least at the precision of our data.

\subsection{Testing the effect of gas kinematics.}

Several authors have proposed that mechanical energy deposited by shocks is a mechanism to increase the heating of nebulae and a possible source of temperature fluctuations (e.g. Peimbert 1995; Peimbert et al. 1991). One of the manifestations of the shocks consists of line broadening or line splitting due to the presence of different kinematical components. Hence, a way to investigate whether the ADF can be related to excitation by shocks is representing it with respect to the full width at half maximum (FWHM) of different emission lines. In Figure 4 we represent the ADF vs. the FWHM of H $\beta$, [O III] $\lambda 4959$ and [N II] $\lambda 6548$. In order to extend the FWHM baseline, we have introduced the points corresponding to 3 slit positions of NGC 5253 analyzed by López-Sánchez et al. (2007). We have corrected the FWHM by the instrumental width. It is possible to note that FWHM are much larger for the extragalactic objects due to large scale gas movements, that are not observed in the Galactic $\mathrm{H}$ II regions. With the available data, we cannot find a clear relation between the ADF and the represented FWHMs. It is obvious that the paucity of points with large FWHM does not permit to conclude any strong statement about this possible relation. Therefore, it is necessary to increase the number of extragalactic objects with good determinations of both quantities for making a suitable exploration.

\subsection{Testing the effect of dust grain heating.}

Another possible effect that can affect the ADF was proposed by Stasińska \& Szczerba (2001) and consists of photoelectric heating of dust grains in the zones near the ionizing star of a PN that increase $T_{\mathrm{e}}$ in the inner zones producing large $t^{2}$ values. This is the case if the nebula is inhomogeneous in density, because the fluctuation in the ionization parameter 


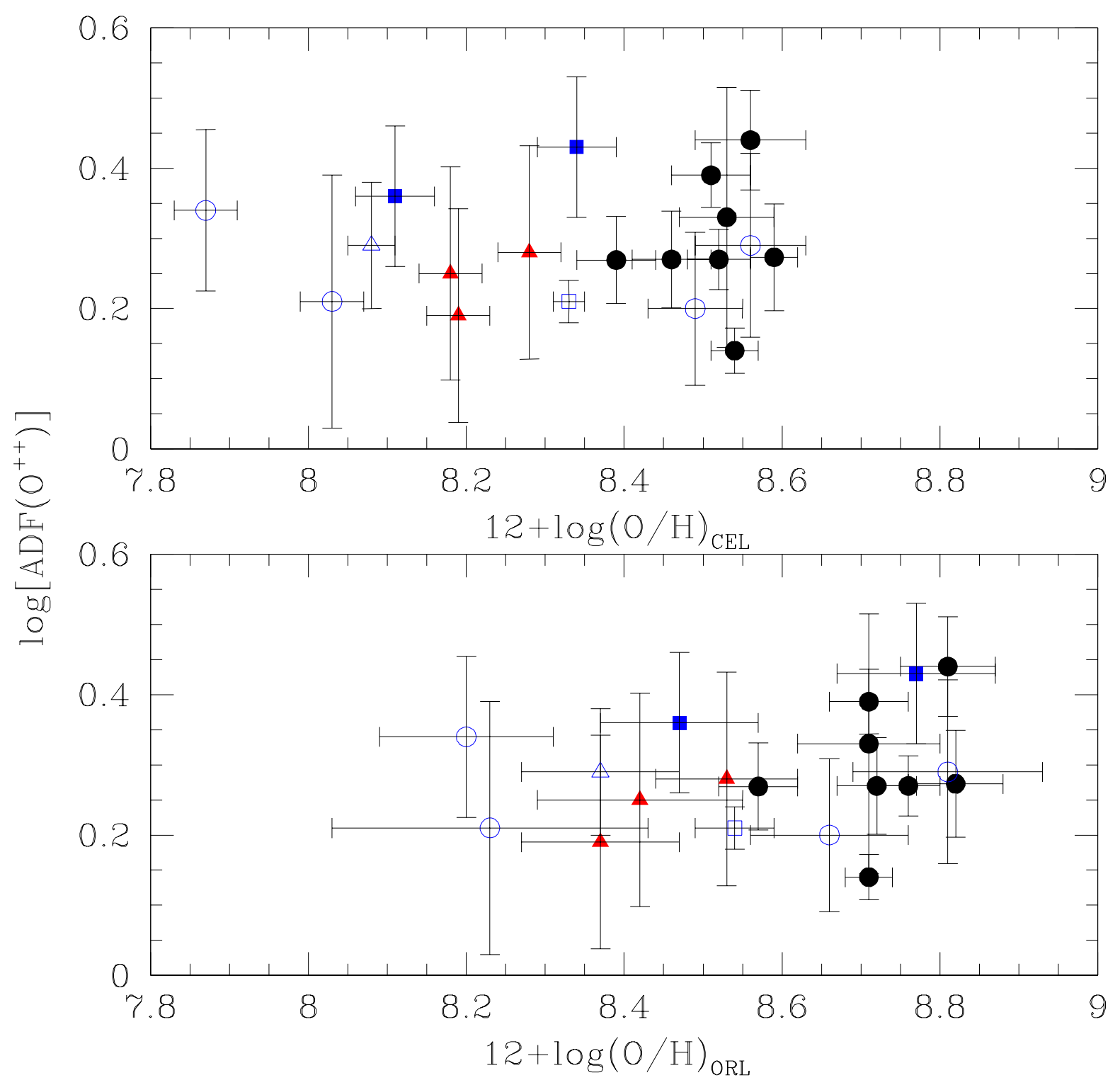

Fig. 2.- $\mathrm{ADF}\left(\mathrm{O}^{++}\right)$vs. total $\mathrm{O}$ abundance derived from CELs (upper panel) and ORLs (lower panel). Symbols are the same that in Figure 1 . 


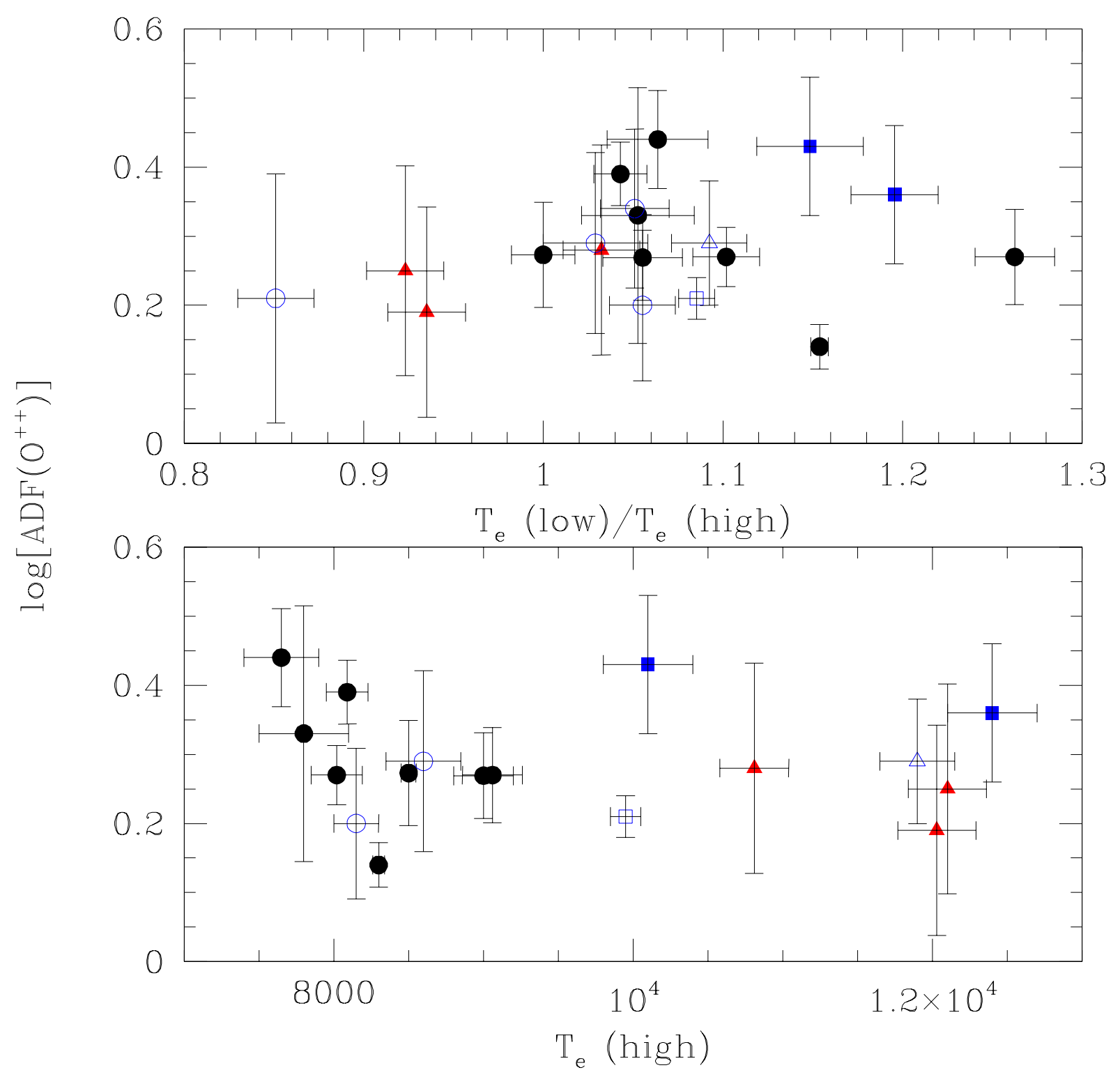

Fig. 3. $-\mathrm{ADF}\left(\mathrm{O}^{++}\right)$vs. $T_{\mathrm{e}}(\mathrm{low}) / T_{\mathrm{e}}$ (high) (upper panel) and vs. $T_{\mathrm{e}}$ (high) (lower panel). Symbols are the same that in Figure 1 . 

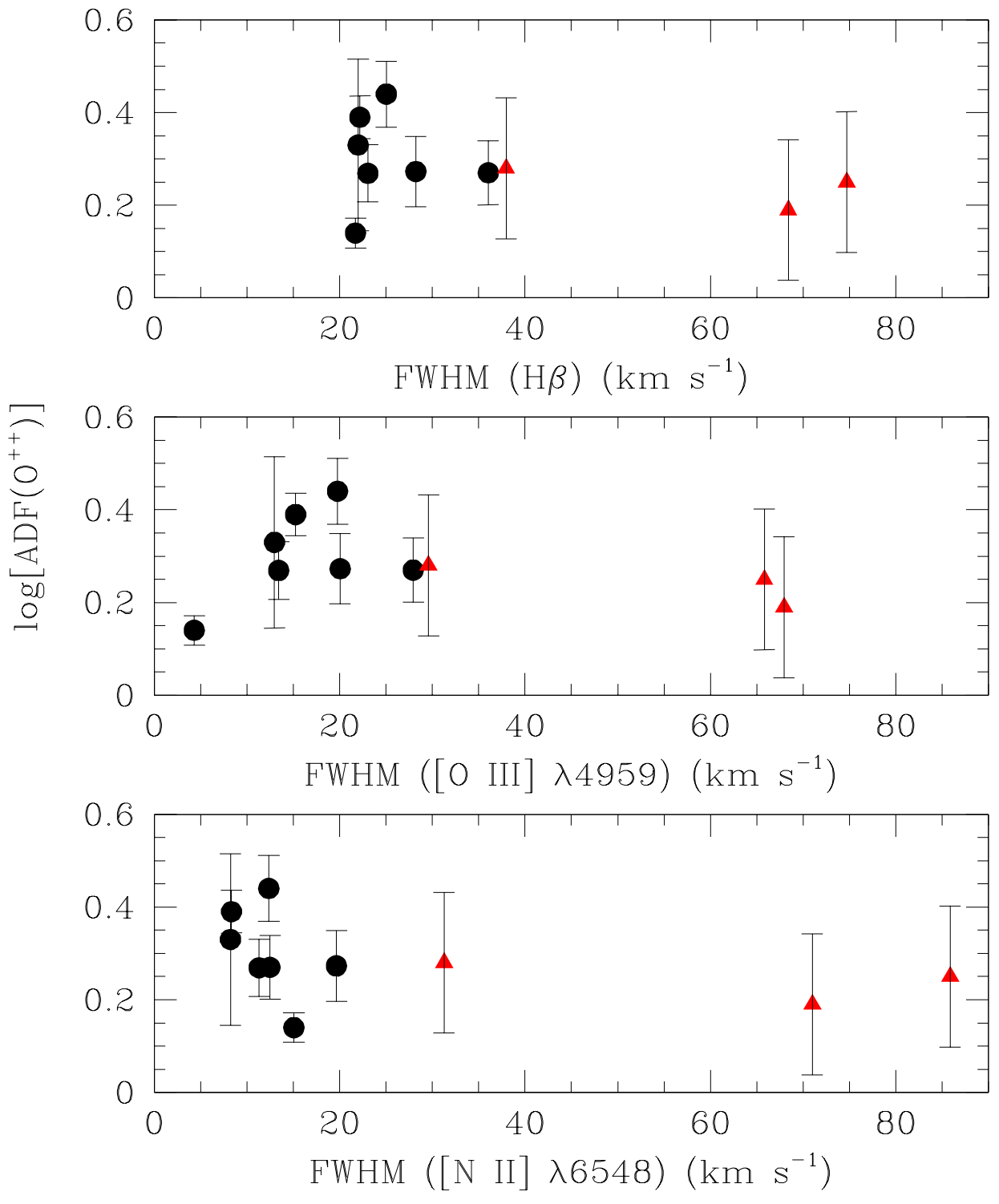

Fig. 4.- $\mathrm{ADF}\left(\mathrm{O}^{++}\right)$vs. FWHM of $\mathrm{H} \beta$ (upper panel), [O III] $\lambda 4959$ (medium panel) and [N II] $\lambda 6548$ (lower panel). Symbols are the same that in Figure 1. 
induced by the variation of density, produce $t^{2}$ due to differential dust and hydrogen heating. In the case of an homogeneous density, dust produces a temperature gradient but not real temperature fluctuations (Stasińska \& Szczerba 2001). A way to verify if the effect of dust grains is significant in the observed ADF is to look for a correlation between the effective temperature, $T_{\text {eff }}$, of the ionizing star of the nebula - that gives an indication of the hardness of the radiation field- and the ADF found in the associated nebula. Robertson-Tessi \& Garnett 2005) obtained that for PNe, the ADF was not correlated with $T_{\text {eff }}$ of the ionizing star. For H II regions this is a complicated task because they are generally ionized by an OB association. In Table 7 we include the $T_{\text {eff }}$ of the main ionizing star (the hottest one) of each studied H II region, and it is clear that such quantity seems to be not correlated with the ADF of the associated nebula.

\subsection{The ADF and recombination continuum/CELs temperature difference.}

The standard temperature fluctuations hypothesis predicts that the ADF is related to the difference between $T_{\mathrm{e}}(\mathrm{CELs})$ and $T_{\mathrm{e}}(\mathrm{H} \mathrm{I})$ (Peimbert \& Costero 1969; Torres-Peimbert

et al. 1980). Indeed, Liu et al. (2000) found a strong correlation between these two discrepancies from data for PNe that cannot be reproduced by the temperature fluctuation paradigm. The results of our work seem to indicate that there is also a slight correlation for H II regions, but with a flatter slope than for PNe (see Figure 5). In Figure 5 we have also included the results obtained for 2 extragalactic H II regions: 30 Doradus (Peimbert 2003) and NGC 2363 (González-Delgado et al. 1994; Esteban et al. 2002). In the case of H II regions, contrary to what happens in $\mathrm{PNe}$, the relation between $\mathrm{ADF}$ and the temperature differences seem to be consistent with the standard temperature fluctuations hypothesis, and they are translated into moderate and similar values of the $t^{2}$ parameter, without reaching the extreme values found in PNe. The fit obtained for our data is:

$$
\begin{aligned}
\log \left[\operatorname{ADF}\left(\mathrm{O}^{++}\right)\right]= & (0.184 \pm 0.022)+(0.609 \pm 0.208) \\
& \times 10^{-4}\left[T_{e}([\mathrm{O} \mathrm{III}])-T_{e}(\mathrm{H} \mathrm{I})\right]
\end{aligned}
$$

with a correlation coefficient of $r=0.575$.

\footnotetext{
${ }^{3}$ For this object the ADF has been obtained from the analysis by Esteban et al. (2002), and temperatures were obtained from González-Delgado et al. (1994). We have included these data because they correspond to the same slit position, covering a very similar volume of nebula.
} 
Table 6. Parameters of the linear least-squares fits of the data represented in Figures 1 to 3 .

\begin{tabular}{cccc}
\hline \hline $\mathrm{ADF}\left(\mathrm{O}^{++}\right)$vs. & Slope & $r$ & $P$ \\
\hline $\mathrm{O}^{+} / \mathrm{O}^{++}$ & 0.099 & 0.204 & 0.411 \\
$12+\log \left(\mathrm{O}^{++}\right) / \mathrm{H}^{+}$ & -0.164 & -0.198 & 0.420 \\
$12+\log (\mathrm{O} / \mathrm{H})_{C E L}$ & -0.049 & 0.064 & 0.768 \\
$12+\log (\mathrm{O} / \mathrm{H})_{O R L}$ & 0.088 & 0.283 & 0.283 \\
$T_{\mathrm{e}}(\mathrm{Low}) / T_{\mathrm{e}}(\mathrm{High})$ & -0.379 & 0.225 & 0.378 \\
$T_{\mathrm{e}}(\mathrm{High})$ & $2.21 \times 10^{-6}$ & -0.113 & 0.646 \\
\hline
\end{tabular}

Table 7. Comparison of the ADF with the spectral type of the main ionizing sources of each of our sample H II regions.

\begin{tabular}{cccc}
\hline \hline Object $^{\mathrm{a}}$ & Star/Cluster & Spectral type $^{\mathrm{b}}$ & $\mathrm{ADF}\left(\mathrm{O}^{++}\right)$ \\
\hline NGC 3603 & NGC 3603/HST-38/40/A2/16 & O3V & 1.9 \\
& NGC 3603/HST-42/A3 & O2-3III & \\
M16 & HD168075 & O4V & 2.8 \\
M17 & Kleinmann's star & O4V & 2.1 \\
M20 & HD164492 & O6V & 2.1 \\
Orión & $\theta^{1}$ Ori C & O6peV & 1.4 \\
S 311 & HD 64315 & O6eV & 1.9 \\
M8 & H36 & O7.5V & 2.0 \\
NGC 3576 & Obscured cluster & OB association & 1.8 \\
\hline
\end{tabular}

${ }^{\text {a } O r d e r e d ~ f r o m ~ e a r l i e r ~ t o ~ l a t e r ~ s p e c t r a l ~ t y p e . ~}$

${ }^{\text {b} F r o m ~ t h e ~ G a l a c t i c ~ O ~ s t a r ~ c a t a l o g ~ b y ~ M a i ́ z-A p e l l a ́ n i z ~ e t ~ a l . ~(2004) . ~}$ 


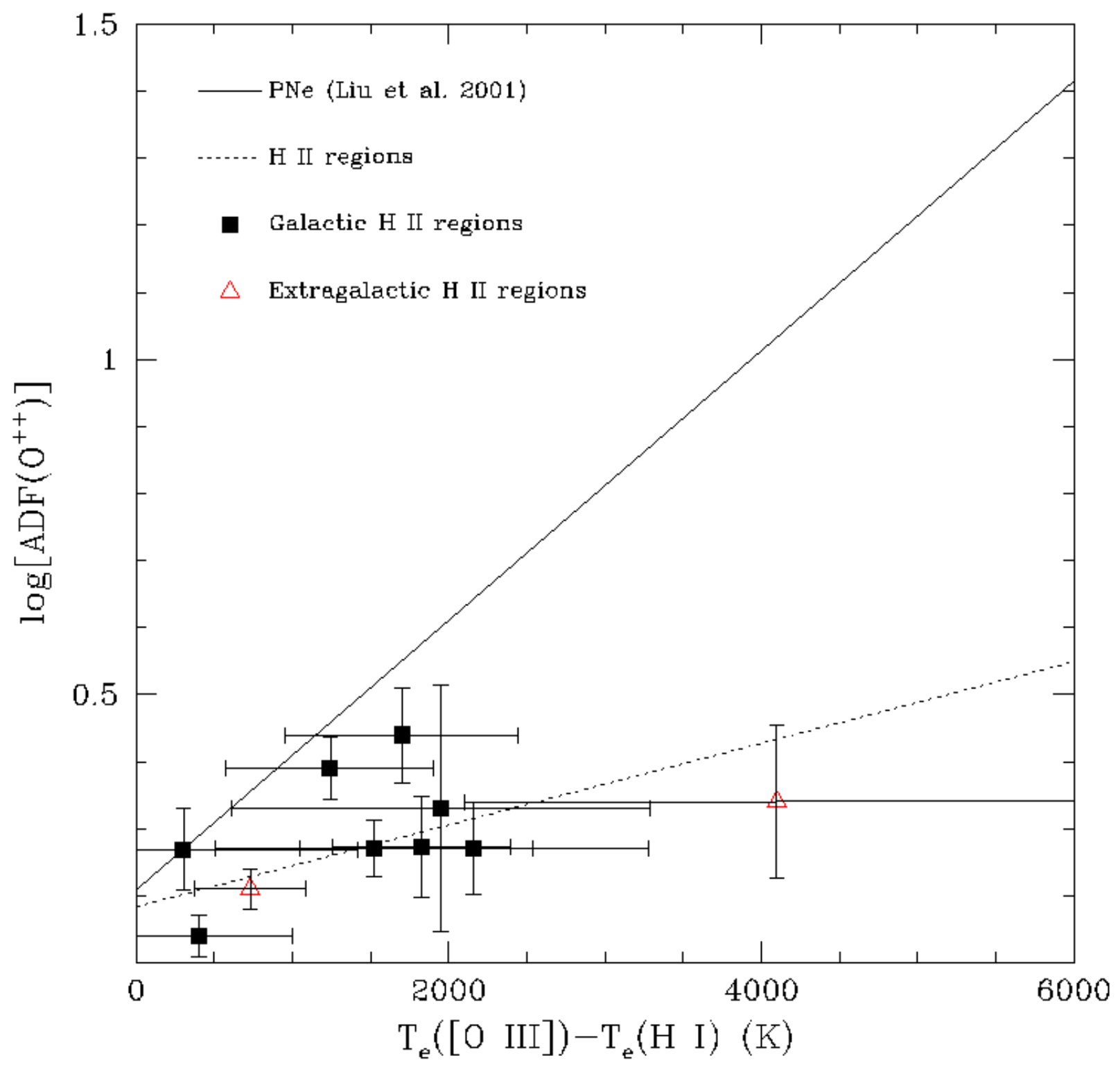

Fig. 5.- $\mathrm{ADF}\left(\mathrm{O}^{++}\right)$vs. the difference between $T_{\mathrm{e}}(\mathrm{CELs})$ and $T_{\mathrm{e}}(\mathrm{H} \mathrm{I})$. Solid line corresponds to the fit found by Liu et al. (2000) for PNe, whereas pointed line corresponds to the fit to our data of H II regions. We have added the data of two extragalactic H II regions: 30 Doradus (Peimbert2003) and NGC 2363 (González-Delgado et al. 1994; Esteban et al. 2002). 
Finally, as we previously pointed out (see García-Rojas et al. 2007), we do not find the correlation between the ADF and the electron density, $n_{\mathrm{e}}$, obtained by Robertson-Tessi \& Garnett (2005) for a PNe sample.

\section{Objections to the two phase model.}

The fact that $\mathrm{H}$ II regions as well as PNe are photoionized nebulae does not imply that photoionization is responsible for all the observed properties of these objects. A common argument against temperature fluctuations in H II regions is based on the difficulties of photoionization models to reproduce $t^{2}$ values of the order of those estimated for most objects. However, photoionization models might not be sufficiently realistic, and/or additional energy sources could be necessary to explain the discrepancies between observations and model predictions (see Viegas 2002, and references therein). Several authors have proposed the existence of a plasma component - colder and metal-rich- (the so-called two-phase model) to explain the dichotomy between abundance and temperature determinations in PNe, where the standard temperature and/or density fluctuations hypothesis do not seem to explain the available observations (Liu et al. 2001; Tsamis et al. 2004). As we have already commented in $\S$ 1. Tsamis \& Péquignot (2005) have proposed a similar explanation for the abundance discrepancy in H II regions although there are not direct observational evidences of the colder and metal-rich inclusions they propose. In the following, we are going to present some objections to this model, based on the results obtained in this work.

In Table 8 we present values of the ADF computed for different ions in Galactic and extragalactic H II regions. The table includes all the data available up to date, including our own results and others from the literature. As we commented before, the ADF for $\mathrm{O}^{++}$ is quite similar in all the objects -Galactic or extragalactic- and also similar to the ADFs for the other ions: $\mathrm{C}^{++}, \mathrm{O}^{+}$, and $\mathrm{Ne}^{++}$. Comparing the results for $\mathrm{H}$ II regions of different galaxies, we find that the ADF seems to be independent of the morphological type, mass, metallicity or even the star formation history of the host galaxy (see also López-Sánchez et a1. 2007). In the case of the Galactic objects, the similarity of the ADFs suggests that the process that produces this phenomenon is independent of the conditions and properties of the Galactic disk, at least along the range of Galactocentric distances covered by our sample. López-Sánchez et al. (2007) have discussed this fact in a paper that presents the ADF values

\footnotetext{
${ }^{3}$ It should be remarked that the hypothesis involved in two phase scenario are in the classical temperature fluctuations paradigm are not of the same order, since the two phase scenario actually gives a natural explanation for the origin of the temperature fluctuations.
} 
for several zones of NGC 5253, a dwarf Wolf-Rayet galaxy.

One of the observational arguments against pure temperature fluctuations in PNe by several authors is the agreement between total abundances derived from optical and FIR CELs in PNe (see e.g. Liu et al. 2001; Tsamis et al. 2004). Due to their low excitation energy, $\mathrm{E}_{e x} \sim 10^{3} \mathrm{~K}$, the fine structure infrared line emissivities have a very weak dependency with $T_{\mathrm{e}}$, therefore they must be insensible to the uncertainties introduced by the presence of temperature fluctuations and, in the case of the existence of such fluctuations, they must give values of abundance similar to that obtained from ORLs. In the case of PNe, this comparison is usually feasible due to the small angular size of these objects, both in the optical range (using scanning techniques to cover the whole nebula, see e.g. Liu et al. 2000), and in FIR and UV (see e.g. Liu et al. 2000, 2001). However, we think that this comparison is not so feasible in the case of extended Galactic H II regions. These objects cover much larger sky areas than PNe, so large uncertainties may arise due to ionization stratification when comparing ionic abundances derived from narrow slit optical spectroscopy and FIR observations obtained with space-borne facilities, that use larger apertures. In a previous paper (García-Rojas et al. 2006) we have showed the difficulties found when comparing our derived total abundances for three objects of our sample (M17, NGC 3603 and the Orion nebula) with those derived from FIR observations (Simpson et al. 1995).

\subsection{Dependence of the ADF on the excitation energy and the critical density.}

By definition, in the temperature fluctuations formulation, the abundance discrepancy must be related to the excitation energy, $\mathrm{E}_{e x}$ of the upper level in which the line originates (see basic equations in Peimbert 1967); whereas in the presence of density fluctuations the abundance discrepancy is maximized if the abundance has been calculated from a CEL coming from a level with low critical density $n_{c}$. This last case implies that the ADF and $n_{c}$ are inversely related. Liu et al. (2000, 2001), comparing different abundance determinations in PNe (UV, optical and FIR CELs), showed that the ADF was not related to $\mathrm{E}_{e x}$ nor to $n_{c}$. We cannot make comparisons between different types of lines (UV, optical or FIR) of the same ion, since we do not have observations of the same zones in other spectral range than the optical one; however, we can compare the ADFs obtained for Galactic and extragalactic $\mathrm{H}$ II regions, with $\mathrm{E}_{e x}$ and $n_{c}$ of the higher level of the main CELs of each ion. In the case of $\mathrm{C}^{++}$the comparison between $\mathrm{UV}$ and optical data could be problematic due to the different volumes of nebula covered by the slits. For NGC 5253, we are sure that

optical and UV observations cover almost the same zones, as was pointed out by LópezSánchez et al. (2007). In the case of M8 and the Orion nebula, the observations do not cover 
Table 8. Observed ADFs in Galactic and extragalactic H II regions.

\begin{tabular}{|c|c|c|c|c|c|c|c|}
\hline ID & $n_{\mathrm{e}}\left(\mathrm{cm}^{-3}\right)$ & $T_{\mathrm{e}}([\mathrm{O} \mathrm{III}])(\mathrm{K})$ & $\operatorname{ADF}\left(\mathrm{O}^{++}\right)$ & $\operatorname{ADF}\left(\mathrm{O}^{+}\right)$ & $\operatorname{ADF}\left(\mathrm{C}^{++}\right)^{\mathrm{a}}$ & $\operatorname{ADF}\left(\mathrm{Ne}^{++}\right)$ & Ref. $^{\mathrm{b}}$ \\
\hline \multicolumn{8}{|c|}{ Galactic H II regions } \\
\hline M16 & 1120 & 7650 & 2.8 & $\ldots$ & $\cdots$ & $\cdots$ & 1 \\
\hline \multirow[t]{2}{*}{ M8 } & 1800 & 8150 & 2.0 & 1.4 & 1.8 & $\ldots$ & 1 \\
\hline & 1750 & 8050 & 2.0 & $\ldots$ & 2.1 & $\ldots$ & 2 \\
\hline \multirow[t]{3}{*}{ M17 } & 470 & 8050 & 2.1 & $4.2:^{\mathrm{c}}$ & $\ldots$ & $\ldots$ & 1 \\
\hline & 860,520 & 8120,8210 & $1.8,2.2$ & $\ldots$ & $\ldots$ & $\ldots$ & 3 \\
\hline & $600-1500$ & 8200 & 2.1 & $\cdots$ & $\cdots$ & $\cdots$ & 4 \\
\hline M20 & 270 & 7980 & 2.1 & 1.5 & $\cdots$ & $\ldots$ & 1 \\
\hline \multirow[t]{2}{*}{ NGC 3576} & 2300 & 8500 & 1.8 & $\ldots$ & $\ldots$ & 2.0 & 1 \\
\hline & $1300-2700$ & 8850 & 1.8 & $\ldots$ & $\ldots$ & $\ldots$ & 4 \\
\hline \multirow[t]{2}{*}{ Orion } & 7800 & 8320 & 1.4 & $1.6^{\mathrm{c}}$ & 1.9 & 1.9 & 1 \\
\hline & 4000,5700 & 8300,8350 & $1.3,1.5$ & $\ldots$ & $2.5,2.2$ & $\cdots$ & 5 \\
\hline NGC 3603 & 3400 & 9030 & 1.9 & $\ldots$ & $\ldots$ & $\ldots$ & 1 \\
\hline S 311 & 310 & 9050 & 1.9 & $\cdots$ & $\cdots$ & $\cdots$ & 1 \\
\hline \multicolumn{8}{|c|}{ Extragalactic H II regions } \\
\hline \multirow[t]{2}{*}{ LMC 30 Dor } & $370-1800$ & 10100 & $2.0-2.7$ & $\ldots$ & $\ldots$ & $\ldots$ & 4 \\
\hline & 316 & 9950 & 1.6 & $\cdots$ & $\cdots$ & $\cdots$ & 6 \\
\hline LMC N11B & $80-1700$ & 9400 & $4.9-8.2$ & $\cdots$ & $\ldots$ & $\cdots$ & 4 \\
\hline SMC N66 & $50-3700$ & 12400 & 2.3 & $\cdots$ & $\cdots$ & $\cdots$ & 4 \\
\hline NGC 604 & $\leq 100$ & 8150 & 1.6 & $\ldots$ & $\ldots$ & $\ldots$ & 7 \\
\hline NGC 2363 & $360-1200$ & 15700 & 2.2 & $\cdots$ & $\cdots$ & $\cdots$ & 7 \\
\hline NGC 5461 & 300 & 8600 & 1.9 & $\ldots$ & $\ldots$ & $\cdots$ & 7 \\
\hline NGC 5471 & $220-1150$ & 14100 & 1.6 & $\cdots$ & $\cdots$ & $\cdots$ & 7 \\
\hline NGC $6822 \mathrm{~V}$ & 175 & 11900 & 1.9 & $\cdots$ & $\ldots$ & $\ldots$ & 8 \\
\hline NGC $5253 \mathrm{~A}$ & 580 & 12100 & 1.8 & $\cdots$ & 2.6 & $\cdots$ & 9 \\
\hline NGC 5253 B & 610 & 12030 & 1.5 & $\ldots$ & 2.1 & $\ldots$ & 9 \\
\hline NGC $5253 \mathrm{C}$ & 370 & 10810 & 1.9 & $\cdots$ & 2.1 & $\ldots$ & 9 \\
\hline
\end{tabular}

${ }^{a}$ CELs abundances for $\mathrm{C}^{++}$were obtained from $I U E$ data of $\mathrm{C}$ III] $\lambda \lambda 1906+1909$ line. Peimbert et al. 1993b; Walter et al. 1992, Kobulnicky et al. 1997, for M8, the Orion nebula and NGC 5253, respectively).

b1) This work; 2) Esteban et al. 1999b; 3) Esteban et al. (1999a); 4) Tsamis et al. (2003); 5) Esteban et al. (1998); 6) Peimbert 2003); 7) Esteban et al. 2002); 8) Peimbert et al. (2005); 9) López-Sánchez et al. 2007 ).

${ }^{\mathrm{c}}$ Dubious value. 
the same zone, but we have compared the ionization correction factor for $\mathrm{C}^{++}$obtained from computing $\mathrm{C}^{+} / \mathrm{H}^{+}$ratio from $I U E$ observations of the UV C II] $\lambda 2326$ line [Peimbert et al. 1993b; Walter et al. 1992, for M8 and the Orion nebula, respectively) and by using the ionization correction factor (ICF) of Garnett et al. (1999). From these data we have obtained ICFs of 2.48 and 2.45 for M8, and 1.20 and 1.24 for the Orion nebula, suggesting that there are not significant changes in the ionization structure of the observed zones in the optical and UV. In Figure 6 it can be noted that, taking into account the uncertainties, a slight correlation $(r=0.7, P=0.188)$ between the $\mathrm{ADF}$ and $\mathrm{E}_{e x}$ is apparent. There is also an apparent correlation $(r=0.7, P=0.188)$ with $n_{c}$, the opposite to that predicted by density fluctuations, which suggests that density fluctuations should play a much less important role than temperature fluctuations in H II regions. These results provide an additional argument that the mechanism that produces the abundance discrepancy in H II regions should be different to that proposed for PNe and, therefore, we can not make the same considerations when working with PNe and H II regions. In Table 9, we show the values of ADF, $\mathrm{E}_{e x}$ and $n_{c}$ adopted for each ion. The values we have adopted are the weighted mean of the ADFs computed for each ion in different objects. We distinguish between $\mathrm{O}^{+}$abundances from [O II] $\lambda \lambda 3726+3729$ and $\lambda \lambda 7320+30$ because they have very different $\mathrm{E}_{e x}$ and $n_{c}$.

\subsection{O II ORLs temperature.}

The two phase model predicts that $T_{\mathrm{e}}$ (ORLs) $\leq T_{\mathrm{e}}$ (He I) $\leq T_{\mathrm{e}}(\mathrm{H} \mathrm{I}) \leq T_{\mathrm{e}}(\mathrm{CELs})$ (Liu 2003), with the difference between any of these two temperatures being proportional to the ADF (see e.g. Figure 5).

Wesson et al. (2003) made use -for the first time- of the temperature sensitive ratio $I(\lambda 4089.29) / I(\lambda 4649.14)$ to determine $T_{\mathrm{e}}$ of the ionized gas in which O II lines arise in PNe. These authors found very low $T_{\mathrm{e}}$ in two H-deficient knots in the PN Abell 30. Later, several authors have found similar results in other PNe (e.g. Tsamis et al. 2004; Liu et al. 2004; Wesson et al. 2005). The intensities of the ORLs that originate from states with different orbital angular momentum present different dependencies with $T_{\mathrm{e}}$. For example, comparing the intensity of one $3 d-4 f$ transition with respect to a $3 s-3 p$ transition, is possible to compute the electron temperature. However, this method has its difficulties: first, the dependency of the intensity ratio with the temperature is weak, so these lines must be measured very accurately; second, the relative intensities of the O II lines can be affected by departures of the local thermodynamic equilibrium (LTE) in the fundamental recombination level of the $\mathrm{O}^{++}$ion, ${ }^{3} \mathrm{P}$. Tsamis et al. (2004) consider the ratio of the $3 d-4 f$ transition of O II $\lambda 4089.29$ and the $3 p-3 s$ transition O II $\lambda 4649.14$ of multiplet 1 because these lines arise from states of 

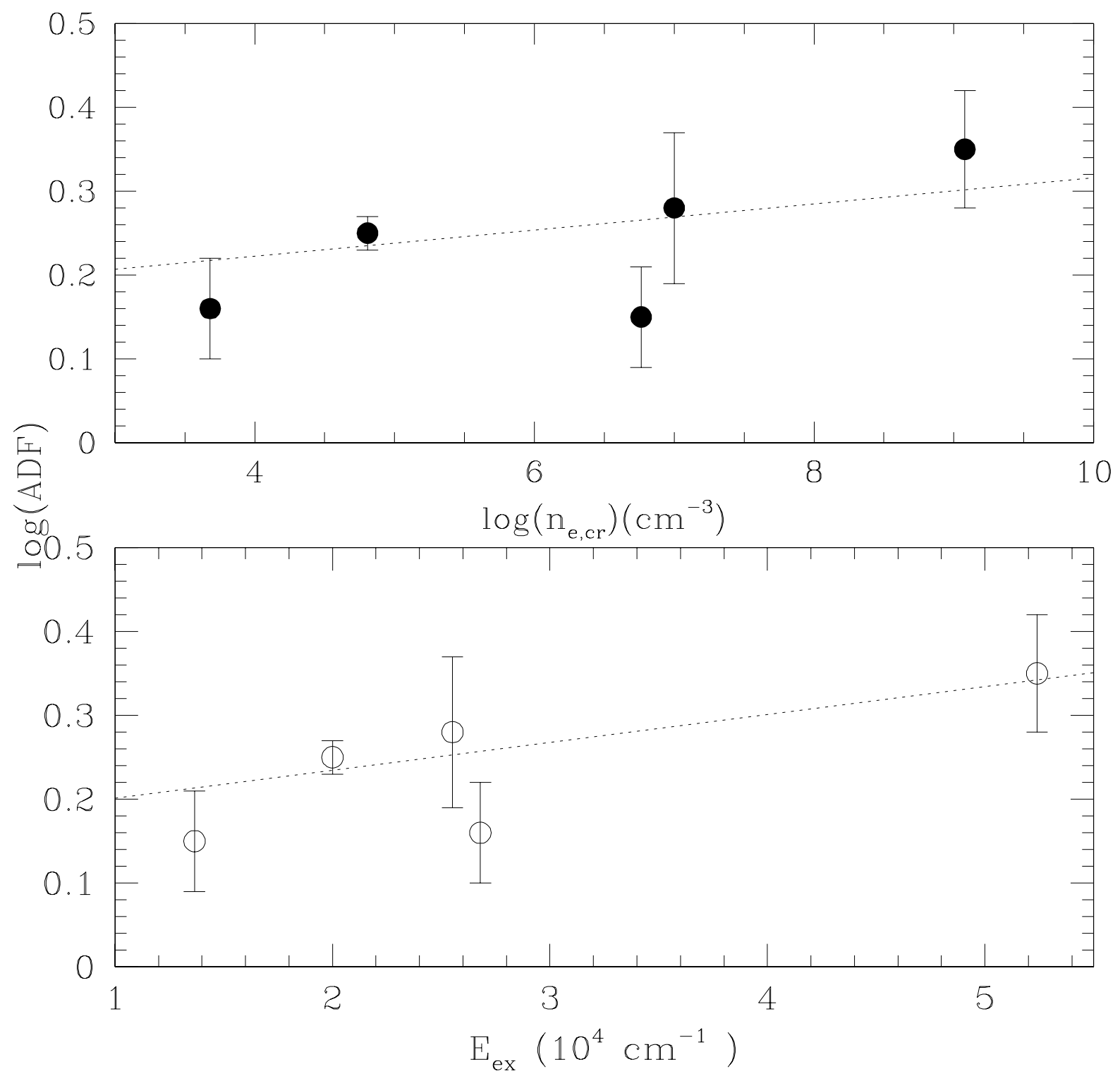

Fig. 6.- ADF (obtained for different ions) vs. excitation energy of the upper level from which each line originates (down) and critical density (up). In upper panel, the represented ions are, from left to right: $\mathrm{O}^{+}([\mathrm{O} \mathrm{II}] \lambda \lambda 3726+29), \mathrm{O}^{++}, \mathrm{O}^{+}(\lambda \lambda 7319+20+29+30), \mathrm{Ne}^{++}$and $\mathrm{C}^{++}$; in lower panel the represented ions are, from left to right: $\mathrm{O}^{+}(\lambda \lambda 7319+20+29+30)$, $\mathrm{O}^{++}, \mathrm{Ne}^{++}, \mathrm{O}^{+}([\mathrm{O} \mathrm{II}] \lambda \lambda 3726+29)$ and $\mathrm{C}^{++}$. 
high total angular momentum $\mathrm{J},{ }^{3} \mathrm{P}_{2}$ and, therefore they must be affected in a similar way by this effect.

Determination of electron temperature from O II ORLs in H II regions is a non trivial problem because in these objects ORLs are usually much weaker than in PNe. In Figure 7 we have represented the change of $I(\lambda 4089.29) / I(\lambda 4649.14)$ ratio with $T_{\mathrm{e}}$ for $T_{\mathrm{e}}=10^{4} \mathrm{~cm}$ ${ }^{-3}$, and we have compared that function with the ratios obtained in NGC 3576 and the Orion nebula, the only objects of our sample where O II $\lambda 4089.29$ line has been measured. Additionally, we have represented the ratio measured in the giant $\mathrm{H}$ II region 30 Doradus in the Large Magellanic Cloud (LMC) (Peimbert 2003) and in several PNe taken from the literature: M 1-42 and M 2-36 (Liu et al. 2001), Tsamis et al. (2004) sample, PNe with $\mathrm{ADF}>4$ of Wesson et al. (2005) sample, NGC 5307 (Ruiz et al. 2003) and NGC 5315 (Peimbert et al. 2004). This last PN is also included in the PNe sample of Tsamis et al. (2004) and we have considered it with the purpose of comparing the effect that high spectral resolution observations introduce in the determination of $T_{\mathrm{e}}(\mathrm{O} \mathrm{II})$. NGC 5315 is appropiate because NLTE effects are small due to their high $n_{\mathrm{e}}\left(\sim 10^{4} \mathrm{~cm}^{-3}\right)$ and also presents an ADF which is similar to that measured in H II regions. Within the sample of PNe included in panel a) of Figure 7 there are objects with very high ADF (between 5.0 and 22) and in panel b) of Figure 7 there are other PNe that show values similar to that obtained in H II regions. NGC 5307 and NGC 5315 show a moderate ADF (1.9 and 1.7 respectively), and have been studied from high resolution spectra $(\mathrm{R} \sim 8800)$, which avoids the overlapping of O II multiplet 1 lines (in the other PNe, O II $\lambda 4649.14$ has been measured deblending the line using a multiple Gaussian fit) and of O II $\lambda 4089.29$ line with other spectral features $\$$. As we can see in panel a) of Figure 8, PNe with high ADFs show, in general, very low $T_{\mathrm{e}}(\mathrm{O}$ II), which is consistent with the two phase model predictions. On the other hand, PNe with moderate ADFs (panel b) of Figure 7) present temperatures that, in general, are also below $T_{\mathrm{e}}(\mathrm{CELs})$ and $T_{\mathrm{e}}(\mathrm{H} \mathrm{I})$, except in the cases of NGC 5882 (Tsamis et al. 2004) and NGC 5315 (Peimbert et al. 2004), where $T_{\mathrm{e}}\left(\mathrm{O}\right.$ II) is consistent with the derived $T_{\mathrm{e}}$ from CELs. On the other hand, $T_{\mathrm{e}}(\mathrm{O}$ II) obtained for NGC 5307 is extremely low, which can be due to uncertainties in the measurement of the intensity of O II $\lambda 4089.29$ line (see Figure 8). In

\footnotetext{
${ }^{4} \mathrm{In}$ some of the PNe of the Tsamis et al. sample, the contribution of Si IV $\lambda 4088.85$ line to the intensity of O II $\lambda 4089.29$ line was corrected using the measured intensity of Si IV $\lambda 4116.10$ line and adopting the theoretical Si IV $\lambda 4088.85 / \mathrm{Si}$ IV $\lambda 4116.10$ flux ratio (2:1).

${ }^{5}$ The NGC 5307 spectrum was obtained in the same observation run as those of NGC 3576 and the Orion nebula. In the analyzed spectra of these observations, O II $\lambda 4089.29$ line is closely blended with a spectral feature produced by charge transfer effects in the CCD due to the presence of a very bright line in a different order; this feature is clearly separated from the O II $\lambda 4089.29$ line in the Orion nebula, but not in NGC 3576 nor in 30 Doradus (see Figure 8). An alternative form to correct it, would be to adopt the theoretical ratio
} 
the case of the Galactic $\mathrm{H}$ II regions, $T_{\mathrm{e}}(\mathrm{O}$ II) obtained for the Orion nebula is somewhat higher to that obtained from CELs; for NGC 3576, $T_{\mathrm{e}}(\mathrm{O}$ II $)$ is smaller than that obtained from CELs, but O II $\lambda 4089.29$ line is affected by charge transfer effects in the CCD (see Figure 8). Similarly, $I(4089) / I(4649)=0.825$ ratio found for 30 Doradus (Peimbert 2003) is extremely high, due to a significant contribution of charge transfer to the intensity of the line (see Figure 8). For these two regions we have tried to separate the line from the parasite feature, but it was not easy because of the low signal-to-noise of the data in the spectral range of interest. As a first step we have identified the line responsible of the charge transfer effect in the CCD and then we have measured the flux of all the features produced by charge transfer in the CCD (hereinafter CCT) in all the orders; finally, we have fitted the flux decrement through the different orders in order to estimate the contribution of the CCT to the O II $\lambda 4089.29$ line. Unfortunately, uncertainties in the observed fluxes were high, so we can only make a rough estimation to the corrected line flux. For NGC 3576, we have estimated that CCT is about a $16 \%$ of the observed flux of the O II $\lambda 4089.29$ line. In Figure $\mathbb{7 c}$ we have represented the new corrected value for NGC 3576, adopting the same uncertainty in the line flux that before correction. In the case of 30 Doradus, we have estimated that the contribution to the observed flux of O II $\lambda 4089.29$ line is between $30 \%$ and $60 \%$. We have represented an intermediate case (correction of $45 \%$ ) in Figure đd, assuming that the uncertainty in the $I(4089) / I(4649)$ ratio of 30 Dor is about $30 \%$ or even greater. As it can be seen in Figure $7 \mathrm{c}$, applying the estimated corrections, the $T_{\mathrm{e}}(\mathrm{O}$ II $)$ obtained for NGC 3576 is now consistent with $T_{\mathrm{e}}$ derived from CELs. For 30 Doradus, taking into account that the $n_{\mathrm{e}}$ derived by Peimbert (2003) was $\sim 300 \mathrm{~cm}^{-3}$, we have represented the theoretical $I(\lambda 4089.29) / I(\lambda 4649.14)$ ratio for $n_{\mathrm{e}}=250 \mathrm{~cm}^{-3}$ (P.J. Storey, private communication) which is quantitatively different from that derived for $n_{\mathrm{e}}=10^{4} \mathrm{~cm}^{-3}$; unfortunately, in this case, the results are not as clear as in the case of NGC 3576: the large uncertainties in the adopted flux of the O II $\lambda 4089.29$ line makes the O II $I(4089) / I(4649)$ ratio unreliable to compute $T_{\mathrm{e}}$ because uncertainties are compatible with variations of $T_{\mathrm{e}}$ from $600 \mathrm{~K}$ to $25000 \mathrm{~K}$ (see Figure $7 \mathrm{~d}$ ). In order to have additional H II region data, we have represented the value of the intensity ratio for non-published UVES datasets for two slit positions of the Orion nebula: the position labeled as "Orion 1" coincides with one of the two zones studied by Esteban et al. (1998), and one slit position located on the Orion bar $\left(24^{\prime \prime} \mathrm{N}\right.$ and $12^{\prime \prime} \mathrm{W}$ of $\theta^{2}$ Ori A). From the position of the error boxes for these two regions in Figure $\mathbb{7} \mathrm{c}$, it can be seen that the values of $T_{\mathrm{e}}$ are similar to that obtained for the Orion 2 slit position. Considering $T_{\mathrm{e}}([\mathrm{O} \mathrm{III}])$ calculated for these two regions and the relatively high densities that have been computed in both regions $-n_{\mathrm{e}}([\mathrm{Cl} \mathrm{III}])=7900 \pm 1300$ and $5300 \pm 800 \mathrm{~cm}^{-3}$ for

with other line of the same multiplet but unfortunately we do not know the effect of NLTE to the relative intensity of the lines of the multiplet. 
Orion 1 and Bar, respectively, which minimizes NLTE effects in the intensity of the lines-, we can affirm that $T_{\mathrm{e}}(\mathrm{O}$ II) measured in different positions from the Orion nebula are similar or even somewhat larger than that measured from CELs. On the other hand, it is true that in the presence of pure temperature fluctuations, $T_{\mathrm{e}}(\mathrm{O}$ II) should be somewhat lower than that measured from CELs but, taking into account that measured $t^{2}$ 's in H II regions are moderate, differences as large as those predicted by the two-phase model are not expected.

Unfortunately, the available data for H II regions are not enough to draw definitive general conclusions; however our analysis allows us to enumerate a series of problems that we have found and to reach some partial conclusions; a) first, it is necessary to develop atomic models that consider departures from LS coupling in NLTE, with the purpose of studying how this effect affects each line; b) it is necessary to carry out very deep high resolution spectrophotometry in $\mathrm{H}$ II regions to extend the catalogue of this type of objects with measured $T_{\mathrm{e}}(\mathrm{O}$ II); c) the few available data for $\mathrm{H}$ II regions behave differently from those of most PNe and do not follow the predictions of the two phase model as given in Tsamis \& Péquignot (2005) for 30 Doradus.

Finally, a similar diagram has been constructed using C II RLs ratios: $I(\lambda 9903) / I(\lambda 4267)$ and $I(\lambda 6462) / I(\lambda 4267)$-that are not affected by NLTE effects- but these ratios are much less temperature sensitive than O II $\lambda 4089.29 / \lambda 4649.14$, and variations between $10^{3}$ to $15 \times 10^{3} \mathrm{~K}$ are of the order of the observed error, so these ratios are not reliable to determine accurately electron temperatures.

\section{Conclusions.}

We have derived the abundance discrepancy factor, ADF, defined as: $\mathrm{O}^{++} / \mathrm{H}^{+}(\mathrm{ORLs}) / \mathrm{O}^{++} / \mathrm{H}^{+}(\mathrm{CELs})$ in eight Galactic H II regions, finding similar values for all of them. Furthermore, these values are similar with the ADF determinations in extragalactic H II regions available in the literature. This is a fairly different behavior from the one shown by the ADF in PNe.

We have found that -within the uncertainties- the ADF is not related to $\mathrm{O} / \mathrm{H}, \mathrm{O}^{++} / \mathrm{H}^{+}$, nor to the ionization degree. As well, it is not related either to the assumed $T_{\mathrm{e}}(\mathrm{High})$, so systematic effects in the abundance determination from CELs can be discarded. Also, the $\mathrm{ADF}$ is not related to the $T_{\mathrm{e}}(\mathrm{Low}) / T_{\mathrm{e}}(\mathrm{High})$ ratio, suggesting that $T_{\mathrm{e}}$ natural gradients in $\mathrm{H}$ II regions do not produce the abundance discrepancies.

In the case of H II regions, contrary to the case of PNe, the ADF seems to be metallicity independent in the studied range of $\mathrm{O}$ abundances. Also, there is no evidence that the ADF is related to the effective temperature of ionizing stars - as should be implied by photoelectric 

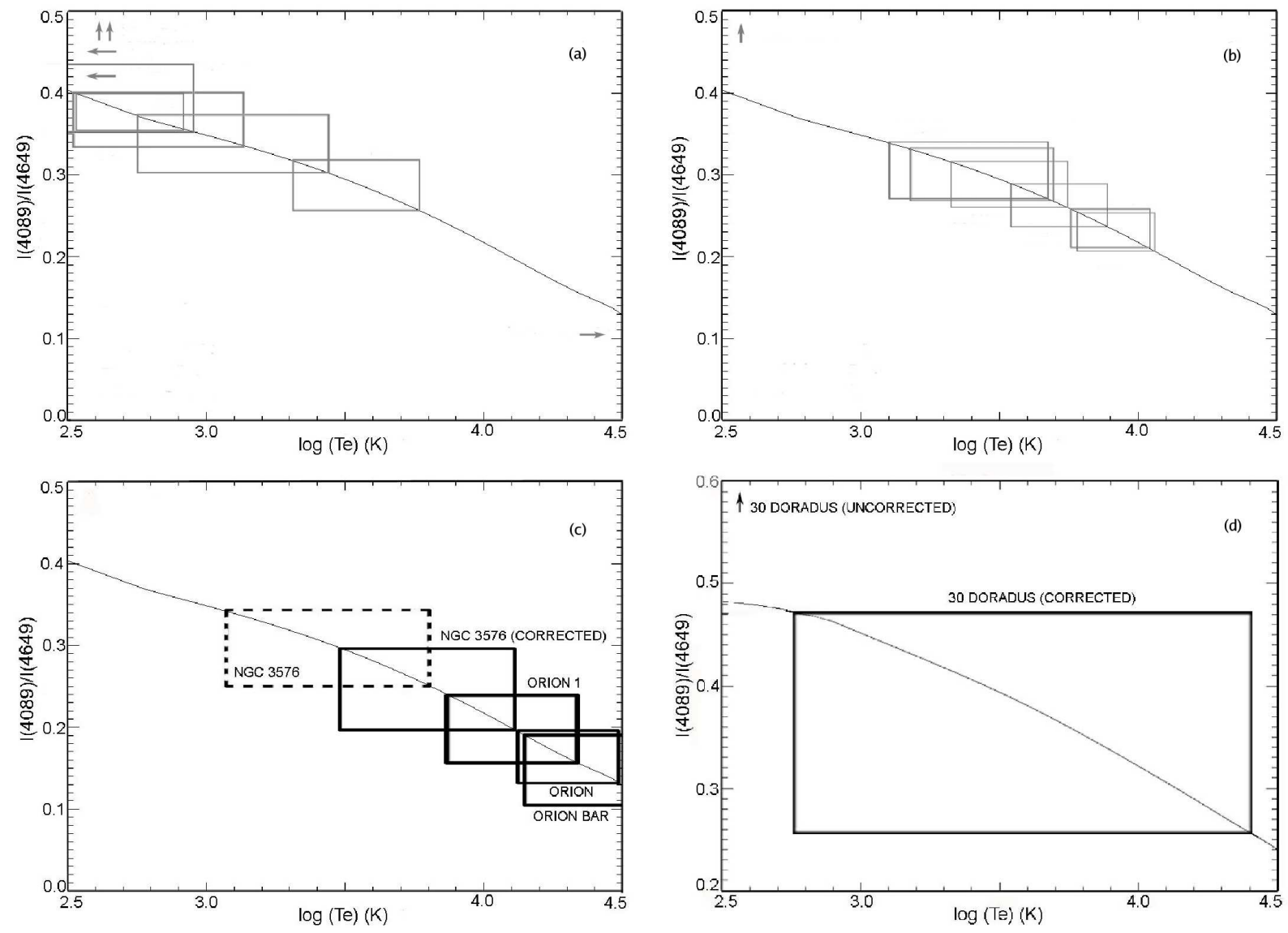

Fig. 7.- O II $\lambda 4089.29 / \lambda 4649.14$ line ratio as a function of the electron temperature. Solid line in a), b) and c) panels is the theoretical ratio for $n_{\mathrm{e}}=10^{4} \mathrm{~cm}^{-3}$. The data are represented as error boxes (see text for the references). In panel a) we have represented a sample of PNe with $\mathrm{ADF}>$ > 5.0; in panel b), we have represented a sample of PNe with moderate ADFs, similar to those found in our sample of H II regions; in the panel c), we show the two $\mathrm{H}$ II regions for which it has been possible to measure O II $\lambda 4089.293 \mathrm{~d}-4 \mathrm{f}$ transition: NGC 3576 and the Orion Nebula and two additional slit positions in the Orion nebula (see text) and, finally, in panel d), we show the data for 30 Doradus (Peimbert 2003). In this last diagram we have also included the theoretical behavior of the line ratio with respect to electron temperature for $n_{\mathrm{e}}=250 \mathrm{~cm}^{-3}$ (P.J. Storey, private communication) in order to perform a more appropiate comparison in the case of 30 Doradus, which shows $n_{\mathrm{e}} \sim 300 \mathrm{~cm}^{-3}$. For NGC 3576 and 30 Doradus, solid line boxes indicate the values found after correction by the presence of charge transfer features in the CCD (see text), the dashed box indicates the position of NGC 3576 before the correction. The horizontal arrows indicate the value of the line ratio for some objects which corresponding temperature is outside the scale, whereas the vertical arrows indicate that the line ratio is outside the represented scale. 

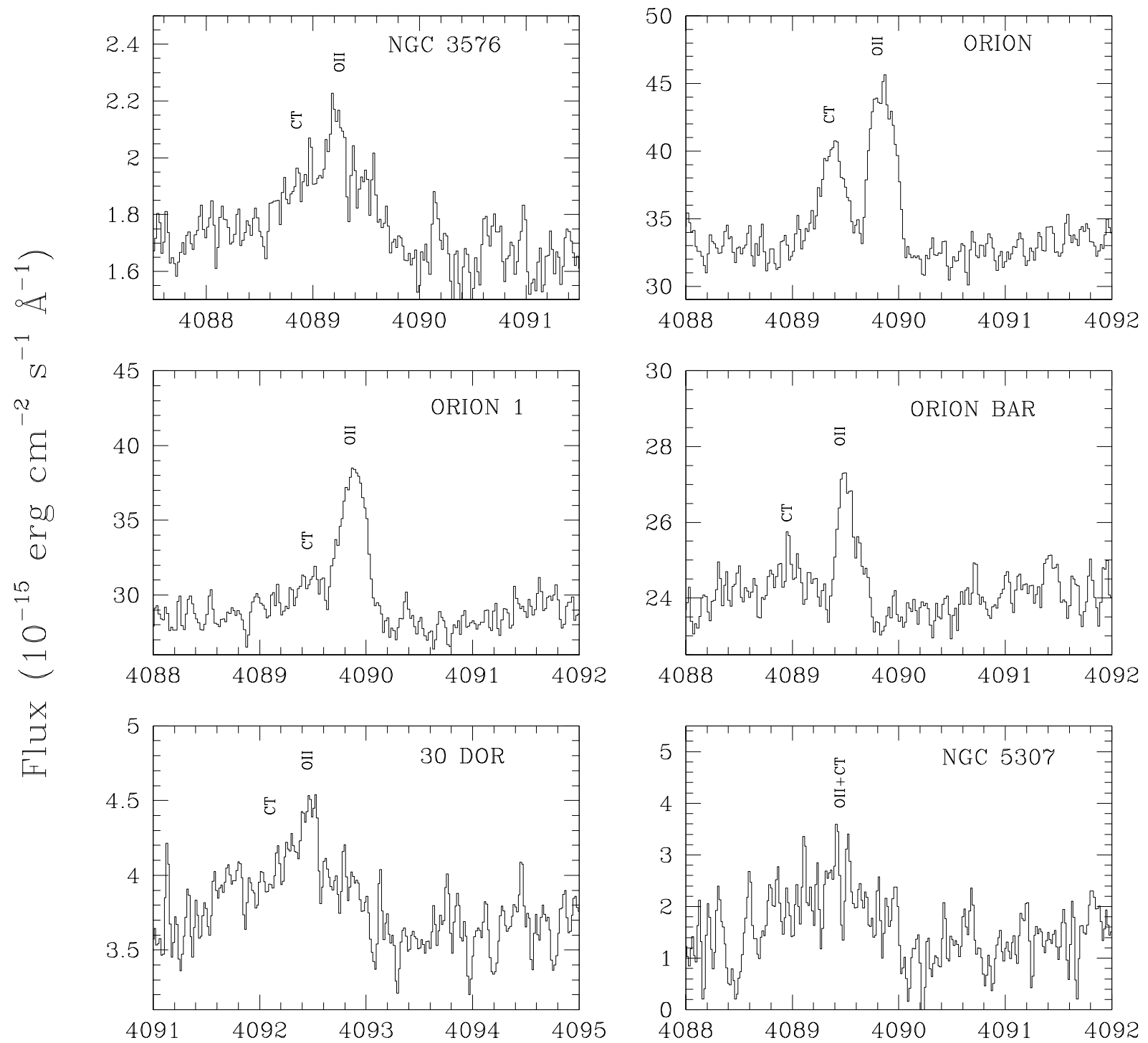

Wavelength $(\AA)$

Fig. 8.- O II $\lambda 4089.19$ line in 5 spectra of $\mathrm{H}$ II regions and in PNe NGC 5307. It can be noticed that this line is severely blended with another feature produced by charge transfer along the CCD (CT) in NGC 3576 and 30 Doradus, whereas in NGC 5307 the signal to noise is too low. The Orion nebula is the only $\mathrm{H}$ II region in which this line has been well measured. The calibrated spectra of 30 Doradus and NGC 5307 have been kindly provided by Antonio Peimbert. 
heating due to the presence of dust near the stars-. We have explored the behavior of the ADF with respect to the FWHM of the spectral lines finding no correlation although the result is not conclusive due to the paucity of objects with lines of large FWHM.

We have found that the ADF seems to be slightly related to the excitation energy, $E_{e x}$ and to the critical density, $n_{c}$ (in contrast to that observed in $\mathrm{PNe}$ ), a behavior that is not predicted by the two phase model initially proposed for PNe and recently extended to H II regions (Tsamis \& Péquignot 2005), but consistent -at least in the first aspect- with the predictions of the standard temperature fluctuations paradigm. On the other hand, electron temperatures derived from ORLs are consistent with those derived from CELs, within the uncertainties, also contradicting the large differences predicted by the two phase model. All these results suggest that the physical mechanism that produces the abundance discrepancy in PNe and H II regions might be different. Our results do not permit to discern what is the mechanism or natural phenomenon that underlies the abundance discrepancy problem, but seem to be more consistent with the predictions of the pure temperature fluctuations paradigm than with those of the two phase model. Therefore, it seems necessary to explore further other alternatives for the origin of the temperature fluctuations and the abundance discrepancy in H II regions.

Obtaining deep spectra of extragalactic H II regions is fundamental to check the different models proposed to explain the abundance discrepancy, permitting to extend the parameters space (metallicity, ionization budget, kinematics, morphological complexity) of the objects.

Finally, we want to stress again that, although the presence and survival of temperature fluctuations in gaseous nebulae is a controversial fact, it is clear that the behavior in H II regions and PNe should be explored in independent ways, due to their different origin, evolution time scales and physical processes that could affect both type of objetcs.

JGR and CE would like to thank M. Peimbert, A. Peimbert, M. Rodríguez, A. Mampaso, V. Luridiana, E. Pérez and P.J. Storey for fruitful discussions and comments. We want also to thank all the organizers and participants to the workshop on Deep Spectroscopy and Modeling of Gaseous Nebulae (held at Xiang Shan, Beijing on Apr 16-18 2007) for fruitful discussions and advice related to this work. We would also thank the referee, G. Stasińska, for her carefully reading of the paper and for her comments, that have increased significantly the quality of the paper. This work has been funded by the Spanish Ministerio de Ciencia y Tecnología (MCyT) under project AYA2004-07466. 


\section{REFERENCES}

Aller, L. H. \& Menzel, D. H. 1945, ApJ, 102, 239

Esteban, C., Peimbert, M., García-Rojas, J., Ruiz, M. T., Peimbert, A., \& Rodríguez, M. 2004, MNRAS, 355, 229

Esteban, C., Peimbert, M., Torres-Peimbert, S., \& Escalante, V. 1998, MNRAS, 295, 401

Esteban, C., Peimbert, M., Torres-Peimbert, S., \& García-Rojas, J. 1999a, Rev. Mexicana Astron. Astrofis., 35, 65

Esteban, C., Peimbert, M., Torres-Peimbert, S., García-Rojas, J., \& Rodríguez, M. 1999b, ApJS, 120, 113

Esteban, C., Peimbert, M., Torres-Peimbert, S., \& Rodríguez, M. 2002, ApJ, 581, 241

García-Rojas, J., Esteban, C., Peimbert, A., Peimbert, M., Rodríguez, M., \& Ruiz, M. T. 2005, MNRAS, 362, 301

García-Rojas, J., Esteban, C., Peimbert, A., Rodríguez, M., Peimbert, M., \& Ruiz, M. T. 2007, Rev. Mexicana Astron. Astrofis., 43, 3

García-Rojas, J., Esteban, C., Peimbert, M., Costado, M. T., Rodríguez, M., Peimbert, A., \& Ruiz, M. T. 2006, MNRAS, 368, 253

García-Rojas, J., Esteban, C., Peimbert, M., Rodríguez, M., Ruiz, M. T., \& Peimbert, A. 2004, ApJS, 153, 501

Garnett, D. R. 1992, AJ, 103, 1330

Garnett, D. R. \& Dinerstein, H. L. 2001, ApJ, 558, 145

Garnett, D. R., Shields, G. A., Peimbert, M., Torres-Peimbert, S., Skillman, E. D., Dufour, R. J., Terlevich, E., \& Terlevich, R. J. 1999, ApJ, 513, 168

González-Delgado, R. M., Pérez, E., Tenorio-Tagle, G., Vílchez, J. M., Terlevich, E., Terlevich, R., Telles, E., Rodríguez-Espinosa, J. M., Mas-Hesse, M., García-Vargas, M. L., Díaz, A. I., Cepa, J., \& Castañeda, H. 1994, ApJ, 437, 239

Guseva, N. G., Izotov, Y. I., Papaderos, P., \& Fricke, K. J. 2007, A\&A, in press, astro$\mathrm{ph} / 0701032$

Guseva, N. G., Izotov, Y. I., \& Thuan, T. X. 2006, ApJ, 644, 890 
Hägele, G. F., Pérez-Montero, E., Díaz, A. I., Terlevich, E., \& Terlevich, R. 2006, MNRAS, 372, 293

Harrington, J. P., Lutz, J. H., Seaton, M. J., \& Stickland, D. J. 1980, MNRAS, 191, 13

Kaler, J. B. 1986, ApJ, 308, 337

Kobulnicky, H. A., Skillman, E. D., Roy, J.-R., Walsh, J. R., \& Rosa, M. R. 1997, ApJ, 477, 679

Liu, X.-W. 2003, in IAU Symp. 209, Planetary Nebulae: Their Evolution and Role in the Universe (San Francisco: ASP), ed. S. Kwok, M. Dopita, \& R. Sutherland, 339

Liu, X.-W. 2006, in IAU Symp. 234, Planetary Nebulae in our Galaxy and Beyond (San Francisco: ASP), ed. M. J. Barlow \& R. H. Méndez, 219

Liu, X.-W., Barlow, M. J., Zhang, Y., Bastin, R. J., \& Storey, P. J. 2006, MNRAS, 368, 1959

Liu, X.-W., Luo, S.-G., Barlow, M. J., Danziger, I. J., \& Storey, P. J. 2001, MNRAS, 327, 141

Liu, X.-W., Storey, P. J., Barlow, M. J., \& Clegg, R. E. S. 1995, MNRAS, 272, 369

Liu, X.-W., Storey, P. J., Barlow, M. J., Danziger, I. J., Cohen, M., \& Bryce, M. 2000, MNRAS, 312, 585

Liu, Y., Liu, X.-W., Barlow, M. J., \& Luo, S.-G. 2004, MNRAS, 353, 1251

López-Sánchez, A. R., Esteban, C., García-Rojas, J., Peimbert, M., \& Rodríguez, M. 2007, ApJ, 656, 168

Maíz-Apellániz, J., Walborn, N. R., Galué, H. Á., \& Wei, L. H. 2004, ApJS, 151, 103

Mathis, J. S., Torres-Peimbert, S., \& Peimbert, M. 1998, ApJ, 495, 328

O’Dell, C. R., Peimbert, M., \& Peimbert, A. 2003, AJ, 125, 2590

Osterbrock, D. E., Tran, H. D., \& Veilleux, S. 1992, ApJ, 389, 305

Peimbert, M. 1995, in The Analysis of Emission Lines, ed. R. Williams \& M. Livio, 165

Peimbert, A. 2003, ApJ, 584, 735 
Peimbert, A. \& Peimbert, M. 2005, in Rev. Mexicana Astron. Astrofis. Conf. Ser., ed. D. Torres-Peimbert \& G. MacAlpine, Vol. 23, 9

Peimbert, A., Peimbert, M., \& Ruiz, M. T. 2005, ApJ, 634, 1056

Peimbert, M. 1967, ApJ, 150, 825

Peimbert, M. \& Costero, R. 1969, Boletin de los Observatorios Tonantzintla y Tacubaya, 5, 3

Peimbert, M., Peimbert, A., Ruiz, M. T., \& Esteban, C. 2004, ApJS, 150, 431

Peimbert, M., Sarmiento, A., \& Fierro, J. 1991, PASP, 103, 815

Peimbert, M., Storey, P. J., \& Torres-Peimbert, S. 1993a, ApJ, 414, 626

Peimbert, M. \& Torres-Peimbert, S. 1977, MNRAS, 179, 217

Peimbert, M., Torres-Peimbert, S., \& Dufour, R. J. 1993b, ApJ, 418, 760

Peimbert, M., Torres-Peimbert, S., \& Luridiana, V. 1995, Rev. Mexicana Astron. Astrofis., 31,131

Peimbert, M., Torres-Peimbert, S., \& Ruiz, M. T. 1992, Rev. Mexicana Astron. Astrofis., 24,155

Robertson-Tessi, M. \& Garnett, D. R. 2005, ApJS, 157, 371

Rodríguez, M. \& Rubin, R. H. 2005, ApJ, 626, 900

Rola, C. \& Stasińska, G. 1994, A\&A, 282, 199

Rubin, R. H., Bhatt, N. J., Dufour, R. J., Buckalew, B. A., Barlow, M. J., Liu, X.-W., Storey, P. J., Balick, B., Ferland, G. J., Harrington, J. P., \& Martin, P. G. 2002, MNRAS, 334, 777

Rubin, R. H., Martin, P. G., Dufour, R. J., Ferland, G. J., Blagrave, K. P. M., Liu, X.-W., Nguyen, J. F., \& Baldwin, J. A. 2003, MNRAS, 340, 362

Ruiz, M. T., Peimbert, A., Peimbert, M., \& Esteban, C. 2003, ApJ, 595, 247

Simpson, J. P., Colgan, S. W. J., Rubin, R. H., Erickson, E. F., \& Haas, M. R. 1995, ApJ, 444,721

Stasińska, G., Tenorio-Tagle, G., Rodríguez, M., \& Henney, W. J. 2007, A\&A, arXiv:astro 
Stasińska, G. \& Szczerba, R. 2001, A\&A, 379, 1024

Tenorio-Tagle, G. 1996, AJ, 111, 1641

Torres-Peimbert, S. \& Peimbert, M. 1977, Rev. Mexicana Astron. Astrofis., 2, 181

Torres-Peimbert, S., Peimbert, M., \& Daltabuit, E. 1980, ApJ, 238, 133

Tsamis, Y. G., Barlow, M. J., Liu, X.-W., Danziger, I. J., \& Storey, P. J. 2003, MNRAS, 338,687

Tsamis, Y. G., Barlow, M. J., Liu, X.-W., Storey, P. J., \& Danziger, I. J. 2004, MNRAS, 353, 953

Tsamis, Y. G. \& Péquignot, D. 2005, MNRAS, 364, 687

Viegas, S. M. 2002, Rev. Mexicana Astron. Astrofis. Conf. Ser., 12, 219

Walter, D. K., Dufour, R. J., \& Hester, J. J. 1992, ApJ, 397, 196

Wesson, R., Liu, X.-W., \& Barlow, M. J. 2003, MNRAS, 340, 253

-. 2005, MNRAS, 362, 424

Wyse, A. B. 1942, ApJ, 95, 356 
Table 9. ADFs, excitation energy and critical density of the CELs used to compute the abundances.

\begin{tabular}{cccc}
\hline \hline Ion & $\log (\mathrm{ADF})^{\mathrm{a}}$ & $\mathrm{E}_{\text {ex }}\left(\mathrm{cm}^{-1}\right)$ & $n_{c}\left(\mathrm{~cm}^{-3}\right)$ \\
\hline $\mathrm{C}^{++}$ & $0.35 \pm 0.07$ & 52400 & $1.2 \times 10^{9}$ \\
$\mathrm{Ne}^{++}$ & $0.28 \pm 0.09$ & 25520 & $1.0 \times 10^{7}$ \\
$\mathrm{O}^{++}$ & $0.25 \pm 0.02$ & 20000 & $6.4 \times 10^{4}$ \\
$\mathrm{O}^{+\mathrm{b}}$ & $0.16 \pm 0.06$ & 26800 & $4.8 \times 10^{3}$ \\
$\mathrm{O}^{+\mathrm{c}}$ & $0.15 \pm 0.06$ & 13660 & $5.8 \times 10^{6}$ \\
\hline
\end{tabular}

${ }^{a}$ Weighted mean for all Galactic and extragalactic H II regions.

${ }^{\mathrm{b}}$ From $[\mathrm{O}$ II $] \lambda \lambda 3726+29$.

${ }^{\mathrm{c}}$ From $[\mathrm{O}$ II] $\lambda \lambda 7319+20+29+30$. 